OPEN ACCESS

Edited by:

In-Kyu Park,

Chonnam National University,

South Korea

Reviewed by:

Shiyong Song,

Henan University, China

Ali Zarrabi,

Sabancı University, Turkey

*Correspondence:

Jae Hyung Park

jhpark1@skku.edu

Specialty section:

This article was submitted to

Nanobiotechnology,

a section of the journal

Frontiers in Bioengineering and

Biotechnology

Received: 01 September 2020

Accepted: 30 October 2020

Published: 19 November 2020

Citation:

Pramod Kumar EK, Um W and

Park JH (2020) Recent Developments

in Pathological pH-Responsive

Polymeric Nanobiosensors for Cancer

Theranostics.

Front. Bioeng. Biotechnol. 8:601586.

doi: 10.3389/fbioe.2020.601586

\section{Recent Developments in Pathological pH-Responsive Polymeric Nanobiosensors for Cancer Theranostics}

\author{
E. K. Pramod Kumar' ${ }^{1}$, Wooram Um ${ }^{1}$ and Jae Hyung Park ${ }^{1,2 *}$ \\ ${ }^{1}$ School of Chemical Engineering, College of Engineering, Sungkyunkwan University, Suwon, South Korea, ${ }^{2}$ Biomedical \\ Institute for Convergence at SKKU (BICS), Sungkyunkwan University, Suwon, South Korea
}

Polymeric nanobiosensors (PNBS) that respond to tumor-related factors, including $\mathrm{pH}$, have shown great potential for disease detection owing to their selectivity and sensitivity. PNBS can be converted into theranostic polymeric nanobiosensors (TPNBS) by incorporating therapeutic cargo, thereby enabling concomitant diagnoses and therapy of targeted diseases. The polymeric compartments in TPNBS play a significant role in the development and therapeutic efficacy of nanobiosensors. Polymers enhance the stability, biocompatibility, and selective or effective accumulation of nanobiosensors at desired pathological sites. The intrinsic $\mathrm{pH}$ sensitivity of either the polymers in TPNBS or the TPNBS themselves provides integrated potentialities such as cogent accumulation of TPNBS at the tumor, augmented tumor penetration, cellular uptake, and theranostic activation, including enhanced bioimaging signals and controlled release of therapeutics. In this review, we summarize recent developments in the design, preparation, and characterization of $\mathrm{pH}$-responsive TPNBS and their ability to behave as efficient in vivo nanotheranostic agents in acidic cancer environments.

Keywords: pH-responsive nanotheranostics, magnetic resonance imaging, ultrasound imaging, photoacoustic imaging, fluorescence imaging, photodynamic therapy, sonodynamic therapy, chemodynamic therapy

\section{INTRODUCTION}

Cancer is a major threat to human health worldwide. According to recent statistics, in 2020, the United States is projected to have 1,806,590 new cancer cases and 606,520 cancer-related deaths (Siegel et al., 2020). The cancer death rate increased until 1991 and then decreased continuously till 2017, resulting in an overall decrease of $29 \%$. This was mainly attributed to the research and development in the field of cancer theranostics. Researchers have devoted significant efforts toward understanding the pathogenesis and properties of cancer in order to develop effective treatments for clinical applications. Compared to healthy tissues, the tumor microenvironment (TME),

Abbreviations: PNBS, polymeric nanobiosensors; TPNBS, theranostic polymeric nanobiosensors; TME, tumor microenvironment; ATP, adenosine triphosphate; ROS, reactive oxygen species; RES, reticuloendothelial system; EPR, enhanced permeability and retention; US, ultrasound; PTT, photothermal therapy; PDT, photodynamic therapy; SDT, sonodynamic therapy; CDT, chemodynamic therapy; MRI, magnetic resonance imaging; USI, ultrasound imaging; PAI, photoacoustic imaging; FI, fluorescence imaging; DOX, doxorubicin; HP, tumor-homing peptide; HA, hyaluronan; HAase, hyaluronidase. 
which is composed of cancer-stromal-immune cells and the extracellular matrix, is characterized by uncontrolled cell proliferation. This high cell growth rate is associated with a modified metabolism and the abnormal development of tumor blood vessels. This results in a reduction in the transport of oxygen and nutrients (Vaupel and Harrison, 2004). Approaches for identifying such characteristics of cancer cells have contributed considerably toward the development of tumorspecific nanotheranostics.

Oxidative phosphorylation is a vital pathway of normal cell metabolism; it releases chemical energy from molecular oxygen bonds through the enzymatic oxidation of nutrients during adenosine triphosphate (ATP) synthesis (Da Poian et al., 2010). Oxygen and nutrient deficiency in tumor tissues urge cells to produce energy for survival through anaerobic glycolysis, which differs from oxidative phosphorylation (Vander Heiden et al., 2009). Warburg et al. (1927) studied the anaerobic glycolysis of glucose in tumor veins and arteries. They found more lactic acid and less glucose in tumor tissues than on the arteries, which served as evidence of the modified metabolism in tumor cells. In addition to lactic acid, tumor cells release chemical energy stored in the phosphoanhydride bonds of ATP via hydrolysis, along with the release of carbon dioxide and protons. Thus, the anaerobic glycolysis, or "Warburg effect," increases the acidity and lowers the $\mathrm{pH}$ of pathological sites compared to that in normal tissues. The extracellular $\mathrm{pH}$ values of healthy tissues and the late endosomes-lysosomes of normal cells are approximately 7.4 and 5-6.5, respectively, whereas those of the tumor tissue and the late endosomes-lysosomes of tumor cells are 6-7 and 4.56.5, respectively (Thews et al., 2006; Swietach et al., 2007; Kumar et al., 2014). These $\mathrm{pH}$ values may vary depending on the tumor; however, they are always lower than those of healthy tissues (Danhier et al., 2010). Given the acidic nature of the TME and the late endosomes-lysosomes of the cells, $\mathrm{pH}$-sensitive cancer imaging and therapy using suitable theranostic nanomedicine agents have been developed (Webb et al., 2011).

Theranostic nanomedicine for cancer primarily involves the use of colloidal nanoparticles (NPs) ranging from 10 to $1000 \mathrm{~nm}$ in size (Muthu et al., 2014). Synthetic and natural polymers are vital constituents of these NPs, within which diagnostic and therapeutic agents (organic or inorganic theranostic agents) are encapsulated, conjugated, absorbed, or entrapped. The resulting theranostic polymeric nanobiosensors (TPNBS) simultaneously diagnose and treat cancer at the cellular level (Peng et al., 2015; Zhao et al., 2016). The polymer components improve aqueous stability and the delivery of theranostic cargoes associated with TPNBS (Kakkar et al., 2017; Kocak et al., 2017). An intravenously administered TPNBS may be sequestered by the reticuloendothelial-system (RES)-rich organs such as the liver and kidney. Research on theranostic nanomedicine allows for the development of TPNBS with a non-immunogenic polymer surface to increase their blood circulation half-life (Deepagan et al., 2013; Schöttler et al., 2016); this, in turn, improves their effective accumulation in leaky vasculature-containing tumors, which are associated with uncontrolled cell proliferation (the EPR effect) (Matsumura and Maeda, 1986). In addition to the passive accumulation of TPNBS in the tumor, resulting from the EPR effect, decorating its surface with tumor-specific targeting ligands, such as peptides, small molecules, antibodies, and aptamers, could further improve its targeted (active) accumulation through ligand-receptor interactions (Danhier et al., 2010; Rosenblum et al., 2018). However, the amount of administered TPNBS that can reach the tumor either via passive or active accumulation is still minimal (Wilhelm et al., 2016). This causes imaging and therapeutic functions to appear throughout the body rather than in the tumor alone, resulting in a poor signal-to-noise $(\mathrm{S} / \mathrm{N})$ ratio for imaging and an increased risk of systemic toxicity. Recent studies have focused on overcoming these issues by developing smart, stimuli-responsive TPNBS, which activate imaging and therapeutic functions only in response to endogenous stimuli (e.g., pH, enzymes, redox [glutathione (GSH)-reactive oxygen species (ROS), and hypoxia] or exogenous stimuli (e.g., ultrasound, light, microwave, radiofrequency, and magnetism) present or applied at targeted tumor sites (Feng Q.H. et al., 2018; Kang et al., 2018). Stimuliresponsive TPNBS are switched "on" only at the tumor tissues and afford the selective and sensitive enhancement of imaging and therapeutic efficacy. In contrast, they remain in the "off" state in normal tissues. These TPNBS with improved imaging and therapeutic efficiency provide valuable information, such as that required for detecting diseases, monitoring disease progression, and evaluating patient response to therapy, by performing simultaneous imaging and treatment.

Stimuli-sensitive nanotheranostics offer advantages over conventional passive or active tumor-targeting strategies. Therefore, different stimuli-responsive nanotheranostic agents have been developed (Alsehli, 2020; Gong et al., 2020; Mi, 2020; Parodi et al., 2020). pH-responsive nanotheranostic agents have gained popularity owing to their ability to activate theranostic functions in response to the $\mathrm{pH}$ difference among the normal cells, TME, and intracellular microenvironment of tumors. A large number of polymeric and polymer-coated inorganic nanomaterials exhibiting different physicochemical properties in response to changes in the $\mathrm{pH}$ have been employed for the preparation of acidic TMEs and intracellular microenvironmentresponsive smart nanotheranostics (Feng Q.H. et al., 2018). $\mathrm{pH}$-sensitive hydrophobic to hydrophilic transitional polymers featuring protonatable groups (Bazban-Shotorbani et al., 2017; $\mathrm{Wu}$ et al., 2018), pH-sensitive cleavable linkers containing polymers (Kanamala et al., 2016; Wu et al., 2018), pH-responsive charge and size convertible polymers (Yang et al., 2018; Dai et al., 2020), and pH-sensitive inorganic core-forming materials (Dong et al., 2016), among others, are being used to fabricate nanotheranostic agents (Table 1).

Conventional cancer treatment methods such as chemotherapy, surgery, and radiotherapy fail to cure cancer completely as they are associated with the impairment of the host immune system, adverse drug reactions, poor patient adherence, and low therapeutic efficiency (Nowak et al., 2002; Couzin, 2008; Chaffer and Weinberg, 2011). Thus far, several pH-responsive smart nanomaterials have been developed to overcome these limitations for chemotherapy. Additionally, several studies have convincingly summarized $\mathrm{pH}$-sensitive drug delivery (Mura et al., 2013; Kanamala et al., 2016; Wang et al., 2018; 
TABLE 1 | Summary of pH-responsive nanotheranostics.

\begin{tabular}{|c|c|c|c|c|}
\hline Operational pH & $\begin{array}{l}\text { Mechanism of pH } \\
\text { sensitivity }\end{array}$ & $\begin{array}{l}\text { pH-responsive } \\
\text { chemical structure }\end{array}$ & Functions & References \\
\hline$\sim 6.8$ & $\begin{array}{l}\text { Cyclization-amide bond } \\
\text { degradation (Kang et al., } \\
\text { 2014) }\end{array}$ & DMMA-PEG & (1) Charge reversal (2) PDT, Immunotherapy & $\begin{array}{l}\text { Yang et al., 2018; } \\
\text { Peng et al., } 2020\end{array}$ \\
\hline$\sim 6.8$ & Imine bonds & Benzoic-imine & (1) Charge reversal (2) PTT & Lei et al., 2017 \\
\hline$\sim 6.8$ & Boronate ester & PBA-Gal & (1) Ligand-reversible shielding (2) PDT & Cao et al., 2019 \\
\hline$\sim 6.0$ & Boronate ester & PBA-DA & $\begin{array}{l}\text { (1) Charge reversal and size reduction (2) PTT, PDT, } \\
\text { Chemo, PAI, FI }\end{array}$ & Cong et al., 2020 \\
\hline$\sim 6.8$ & $\begin{array}{l}\text { Cyclization-amide bond } \\
\text { degradation }\end{array}$ & DMMA-PEG & $\begin{array}{l}\text { (1) Charge reverse and size reduction (2) Chemo } \\
\text { and immunotherapy }\end{array}$ & Dai et al., 2020 \\
\hline$\sim 6.8$ & Peptide folding & Melittin peptide & (1) Morphology switching (2) PTT & Jia et al., 2019 \\
\hline$\sim 5.0-6.7$ & CaP degradation & $\mathrm{CaP}$ & (1) Contrast agent $\left(T_{1}\right)$ release (2) MRI & Mi et al., 2016 \\
\hline$\sim 5.5-7.0$ & Structural transformation & i-Motif DNAs & (1) Contrast agent conversion $\left(T_{2}\right.$ to $\left.T_{1}\right)$ (2) MRI & Lu et al., 2018 \\
\hline$\sim 5.0$ & Pore formation & FA-FRT-PFP & (1) Droplet vaporization (2) USI & Li et al., 2020 \\
\hline$\sim 5.5$ & Protonation & $\mathrm{pH}-\mathrm{BDP}$ & (1) Ratiometric PAl of $\mathrm{pH}$ & Miao et al., 2016 \\
\hline$\sim<7.0$ & Protonation & BSA-PANI & (1) Amplified PAl & Tian et al., 2019 \\
\hline$\sim 6.8$ & FRET & ANNA-Cy5.5 & (1) Dual ratiometric $\mathrm{FI}$ of $\mathrm{pH}$ & Ma et al., 2018 \\
\hline$\sim 4.5-5.0$ & Porosity, protonation & $\mathrm{NEt}_{2} \mathrm{Br}_{2} \mathrm{BDP}$ & (1) Amplified FI & Tian et al., 2015 \\
\hline$\sim 6.0-6.2$ & Protonation & mPEG-b-PDPA-Cy7.5 & (1) Augmented PDT and FI & Qi et al., 2019; Yang et al., 2019 \\
\hline$\sim 6.0$ & $\begin{array}{l}\text { Cyclization-amide bond } \\
\text { degradation }\end{array}$ & DEX-MMfu & $\begin{array}{l}\text { (1) Enhanced permeability of TME (2) PAI, PDT, and } \\
\text { Immunotherapy }\end{array}$ & Wang et al., 2019 \\
\hline$\sim 4.3-5.8$ & Degradation & $\mathrm{CaCO}_{3}$ & (1) Bursting effect (2) USI, SDT & Feng Q. et al., 2018 \\
\hline$\sim 6.8$ & Degradation & $\mathrm{MnO}_{2}$ & (1) $\mathrm{O}_{2}$ release (2) MRI, SDT & Lin et al., 2020 \\
\hline$\sim 6.8$ & Degradation & MnS & (1) $\mathrm{MRI}$ and CDT agent release (2) $\mathrm{H}_{2} \mathrm{~S}$ release & He et al., 2020 \\
\hline$\sim 4.4-5.4$ & Degradation & $\mathrm{MnO}_{\mathrm{x}}$ & (1) New mechanism of CDT (2) FI & Lu et al., 2020 \\
\hline
\end{tabular}

Deirram et al., 2019). The development of minimally invasive or non-invasive therapeutic modalities [such as light, ultrasound (US), and endogenous ROS] and induced therapies [such as photothermal-photodynamic therapy (PTT-PDT) (Jaque et al., 2014; Lucky et al., 2015), sonodynamic therapy (SDT) (You et al., 2016), and chemodynamic therapy (CDT) (Tang et al., 2019)] are currently being used in theranostic nanomedicine for cancer treatment. These treatment protocols only damage cancer cells, while leaving normal cells unaffected and intact. The non-invasive therapeutic efficacy can be improved by developing $\mathrm{pH}$-responsive nanotherapeutic agents.

In recent years, $\mathrm{pH}$-responsive $\mathrm{PNBS}$ offering magnetic resonance imaging (MRI), photoacoustic imaging (PAI) fluorescence imaging (FI), ultrasound imaging (USI), and capabilities of improved signal-to-noise ratios in tumors have been developed (Kumar et al., 2013; Kaittanis et al., 2014; Ling et al., 2014; Søndergaard et al., 2015; Wang X. et al., 2015; Wang et al., 2017; Mi et al., 2016; Miao et al., 2016; Zhang et al., 2017b; Vidallon et al., 2020). Therapeutic cargo-incorporated PNBS (TPNBS), which are capable of simultaneous pH-responsive imaging and therapy, have also attracted significant research attention (Wang et al., 2013; Li et al., 2017; Feng Q.H. et al., 2018; Zhu et al., 2018; He et al., 2020). In this review, we evaluate how the development of $\mathrm{pH}$-responsive multifunctional nanomaterials has contributed toward improvements in cancer treatment using the capabilities of these sensors to increase tumor accumulation, tumor penetration, cellular uptake, and theranostic functions. We summarize important recent developments in the design and fabrication of (1) $\mathrm{pH}$-responsive charge and size convertible polymeric nanomaterials for augmented non-invasive cancer therapy, (2) pH-sensitive PNBS for disease detection and bioimaging, and (3) $\mathrm{pH}$-responsive TPNBS for image-guided non-invasive cancer therapy.

\section{pH-RESPONSIVE CHARGE AND SIZE CONVERTIBLE NPS FOR AUGMENTED NON-INVASIVE CANCER THERAPY}

The treatment outcomes of theranostic nanomedicine primarily depends on prolonged in vivo blood circulation, intratumoral penetration, and the subsequent tumor cell internalization of the NPs (Alexis et al., 2008). Rapid clearance of circulating NPs during systemic delivery via RES is a critical issue in nanomedicine. Therefore, it is necessary to minimize the factors that reduce the blood circulation half-life and biodistribution of NPs while designing for theranostic applications. The NP-RES interactions are influenced by factors such as NP composition, size, surface charge, stability, and surface modifications (pegylation and targeting ligands). $\mathrm{pH}$-responsive charge and size-tunable theranostic NPs have been developed to minimize interactions, thereby improving therapeutic efficacy in the tumor. These NPs enhance blood circulation time, selective tumor accumulation, penetration, and cellular internalizations.

\section{pH-Responsive Charge Convertible NPs}

It is well known that NPs with neutral or negatively charged surfaces prevent RES clearance and lengthen the blood 

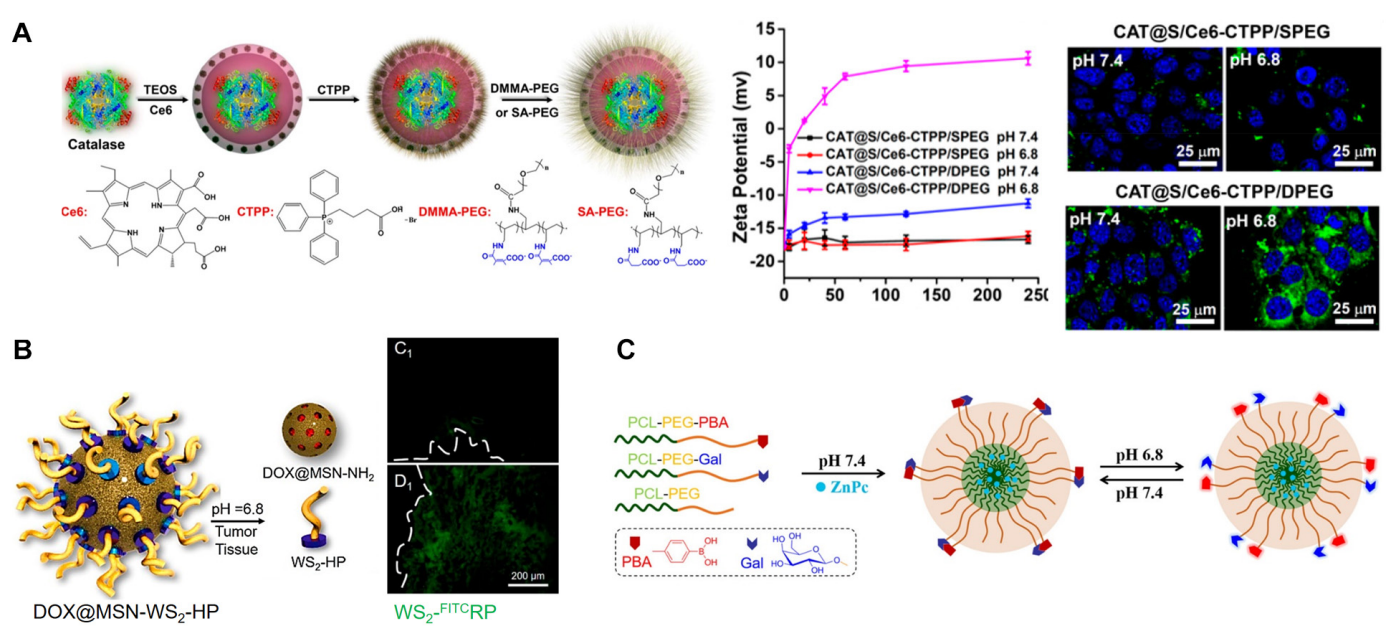

C

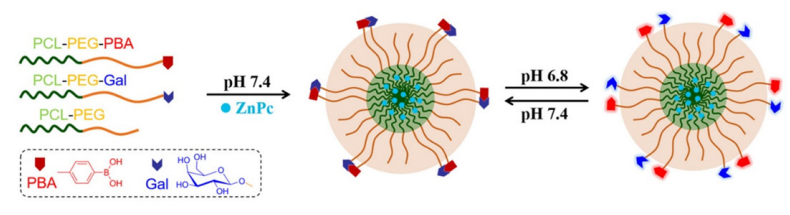

FIGURE 1 | (A) TME pH-responsive charge reversing NPs for enhanced intracellular mitochondrial targeting and improved PDT. Reproduced with permission from reference (Yang et al., 2018). Copyright 2018, American Chemical Society. (B) TME pH-responsive degradation cluster bomb and enhanced tumor penetration of the released $\mathrm{WS}_{2}-{ }^{\mathrm{FITC}} \mathrm{HP}$ quantum dots compared to its control $\left(\mathrm{WS}_{2}-{ }^{\mathrm{FITC}} \mathrm{RP}\right.$ ), confirmed via ex vivo imaging (the dotted line represents the edge of tumor sections). Reproduced with permission from reference (Lei et al., 2017). Copyright 2017, American Chemical Society. (C) pH-responsive ligand-reversible-shielding micelle for enhanced cellular uptake with the associated augmented PDT. Reproduced with permission from reference (Cao et al., 2019). Copyright 2019, American

Chemical Society.

circulation half-life, leading to effective accumulation in tumor tissues owing to the EPR effect. However, a positive surface charge on the NPs would rapidly clear them from the blood through the RES (Yuan et al., 2012; Feng et al., 2014). This positive surface charge, however, facilitates intra-tumoral penetration and the ensuing tumor cell internalization of the NPs (Cho et al., 2009). Therefore, NPs with swappable surface charges that are neutral or negative during blood circulation and quickly convert to positive charges on reaching the acidic TME have recently been annotated as a promising strategy for $\mathrm{pH}$-responsive augmentation in therapy (Han et al., 2016; Zhang et al., 2020).

The non-immunogenic polymer, such as polyethylene glycol (PEG), shielding on NPs creates an inert NP surface that enhances their blood circulation half-life and passive tumor accumulation (Fam et al., 2020). Although the dense PEG corona on NPs affords improved tumor accumulation, the PEGylated NPs exhibit reduced intratumoral penetration, succeeding tumor cell internalization, and endosomal escape (Mishra et al., 2004; Kong et al., 2019). Therefore, significant research efforts have devoted toward designing TME $\mathrm{pH}$-responsive $\mathrm{PEG}$ corona deshielding NPs; the removal of the PEG corona converts surface charges into positive charges, thereby improving the intratumoral penetration and tumor cell internalization of these NPs. Yang et al. (2018) designed a pH-responsive PEG corona with smart nanoreactors for mitochondrial targeting and enhanced photodynamic-immuno combination therapy. Water-soluble, $\mathrm{H}_{2} \mathrm{O}_{2}$-decomposing, and oxygen-producing enzyme catalase (CAT)-encapsulated and photosensitizer (Ce6)loaded hollow silica NPs (S) were prepared (Figure 1A). The NP surface was modified using the mitochondria-targeting molecule 3-carboxypropyl-triphenylphosphonium bromide (CTPP), and the resulting cationic surface was further modified with a pH-responsive charge convertible corona forming anionic PEG 2,3-dimethylmaleic amide (DMMA) polymer (PEG-DMMA) via electrostatic interaction. The obtained CAT@S/Ce6-CTPP/D-PEG featured a well-defined hollow structure with a negative surface charge at neutral $\mathrm{pH}$ because of the PEG shielding. After effective accumulation of the longcirculating NPs in the TME, due to the TME acidity, PEG was detached from the NPs owing to the $\mathrm{pH}$-sensitive cyclizationinduced degradation of the PEG-DMMA amide linkage. This detachment results in a CTPP-exposed cationic NP surface, enabling the effective intracellular targeting of mitochondria, thereby augmenting PDT-induced cancer cell destruction, in comparison with control NPs (CAT@S/Ce6-CTPP/S-PEG) with $\mathrm{pH}$-inert PEG-succinic anhydride (SA-PEG). In the intracellular microenvironment, the catalase encapsulated within these NPs acts as a nanoreactor to trigger the decomposition of tumor-rich endogenous $\mathrm{H}_{2} \mathrm{O}_{2}$, production of $\mathrm{O}_{2}$ used to relieve tumor hypoxia, and further augmentation of in vivo PDT efficacy. A combination of augmented PDT with checkpoint blockade immunotherapy and programmed death-ligand 1 (PD-L1) antibody significantly boosted the infiltration of cytotoxic $\mathrm{T}$ lymphocytes (CTLs) into remote tumors. It impeded their growth, demonstrating a robust abscopal effect encouraging metastasis inhibition. Similarly, $\mathrm{pH}$-responsive charge reversing NPs featuring enhanced tumor targetability and mitochondrial accumulation for PD-immunotherapy were reported recently (Peng et al., 2020). Sequential-targeting positively charged micelles with a Ce6 loaded core were prepared from amphiphilic poly (beta-amino ester), composed of hydrophilic CTTP-PEG and hydrophobic thioketal polymer blocks. The micelles were coated with an anionic $\mathrm{pH}$ responsive charge-transforming shell-forming polymer with tumor-targeting capability (BiotinPEG 4000 -DMMA) using electrostatic interaction. Additionally, $\mathrm{pH}$-responsive charge 
conversion was used to augment mitochondrial targeting, thereby improving PDT efficacy.

Lei et al. (2017) prepared a TME pH-responsive "Cluster Bomb" composed of a drug (DOX) containing loaded mesoporous silica NPs (MSN) capped with tumor-homing or tumor-penetrating peptide tLyP-1-modified tungsten disulfide quantum dots $\left(\mathrm{WS}_{2}-\mathrm{HP}\right)$, by using $\mathrm{pH}$-sensitive benzoic-imine bonds for programmed tumor therapy. The prepared DOX@MSN-WS 2 -HP with the surface-decorated peptide tLyP-1 promotes the tumor-homing ability of 4T1 (Figure 1B). The benzoic-imine bonds between MSN and the quantum dots remain stable during blood circulation. However, they become highly unstable when the TME $\mathrm{pH}$ is 6.8. Once the cluster bomb is accumulated in the TME, it breaks into two parts: DOX@MSN-NH 2 and tLyP-1weraing $\mathrm{WS}_{2}$-HP. Charge-converted electropositive DOX@MSN$\mathrm{NH}_{2}$ enabled efficient chemotherapy on surface tumor cells. The deep tumor-penetrating $\mathrm{WS}_{2}-\mathrm{HP}$ showed tumor suppression in the deep-seated tumor cells by near-infrared (NIR)-light-triggered PTT.

The Yuan group recently reported another interesting strategy of $\mathrm{pH}$-sensitive "ligand-reversible-shielding micelle" for increased tumor accumulation associated with effective PDT (Cao et al., 2019). Phenylboronic acid-functionalized poly(ethylene glycol)- $b$-poly( $\varepsilon$-caprolactone) (PBA-PEG-PCL), and galactose-functionalized diblock polymer (Gal-PEG-PCL) were mixed to prepare dual-ligand micelles (PBA/Gal) to demonstrate this concept (Figure 1C). The PBA and Gal residues form boronate ester complexes at a $\mathrm{pH}$ of 7.4, which mutually shield their targeting function. In an acidic TME ( $\mathrm{pH}$ 6.8), the binding affinity between the PBA and Gal is weakened, and the PBA prefers to bind with the sialic acid residues expressed on the tumor cell surface rather than the Gal on the micellar surface. Furthermore, the unbound Gal recovers its targeting ability toward the asialoglycoprotein receptor, leading to an enhancement in cellular uptake through receptormediated endocytosis. A photosensitizer, zinc phthalocyanine $(\mathrm{ZnPc})$, was loaded onto the reversible-shielding micelle to augment the PDT, owing to its $\mathrm{pH}$-responsive enhanced cellular uptake.

\section{pH-Responsive Size Convertible NPs}

It has been reported that NPs with a size of 10-100 nm and suitable surface modifications achieve efficient tumor accumulation during systemic administration via the EPR effect. NPs smaller than $10 \mathrm{~nm}$ have shown rapid clearance via renal excretion, resulting in reduced tumor accumulation (Cabral et al., 2011; Blanco et al., 2015; Hoshyar et al., 2016). However, compared to large NPs $(10 \mathrm{~nm}<)$, small NPs $(10 \mathrm{~nm}>)$ have shown improved capabilities of intratumoral penetration and subsequent tumor cell internalization (Blanco et al., 2015). Therefore, to solve the size-related conflict issues of NPs, TME or intracellular $\mathrm{pH}$-responsive size swappable NPs have been developed (Sun et al., 2014; Wang J. et al., 2015). In acidic tumors, large NPs accumulated via the EPR effect could undergo a $\mathrm{pH}$-responsive size shrinkage or degradation into smaller NPs. This size reduction improves tumor penetration and cell internalization, resulting in augmented therapeutic efficacy ( $\mathrm{Li}$ et al., 2015; Li H.-J. et al., 2016).

Cong et al. (2020) reported hyaluronan (HA), stacked self-assembled NPs with folic acid (FA) and HA-mediated active targeting capabilities, upon $\mathrm{pH}$-responsive intracellular transformations of size and surface charge. These transformations simultaneously augment tumor penetration, internalization, and intracellular drug release, as well as synergistic photo-chemo combination therapy. They prepared an amphiphilic hexadecapeptide decorated with three cholic acids (CA), one indocyanine green derivative (ICGD), and one 4-carboxy-3-fluorophenylboronic acid (PBA) (Figure 2A). This amphiphilic polypeptide was self-assembled into positively charged small and stable micelle NPs ( $<30 \mathrm{~nm}$ ) (ICP NPs) that are loaded with the antineoplastic drug (SN-38) with a critical micelle concentration (CMC) value of $0.04 \mu \mathrm{M}$, indicating high thermodynamic stability. The ICP NPs were then stacked using dopamine (DA), and folic acid (FA) decorated with HA (HA-DA-FA). This resulted in the formation of large $(130 \mathrm{~nm})$, negatively charged hICP NPs formed through the development of pH-responsive boronate ester bonds between the DA of the HADA-FA and the PBA surface wearing the ICP NPs. Additionally, electrostatic interactions between the negatively charged HA and the positively charged ICP NP surface further strengthens NP stacking. The non-immunogenic HA surface provides improved blood circulation time for the hICP NPs and subsequently accumulates at the TME of subcutaneous B16 melanoma-bearing mice. The hICP NPs were taken up by cancer cells via HA- and FA-mediated endocytosis. At the intracellular level, the hICP NPs underwent size and surface charge transformations in the presence of the enzyme hyaluronidase (HAase) in an acidic $\mathrm{pH}$ in the endosomes/lysosomes; they released small ICP NPs and accelerated intracellular drug release. The transformed ICP NPs facilitated transcytosis-mediated penetration within the tumors. The ICGD in the ICP NPs not only provides NIR light-induced PDT-PTT but also photoacoustic and NIR imaging capabilities. Furthermore, synergistic augmentation of chemo-photo therapeutic efficacy was achieved owing to the $\mathrm{pH}$-responsive size and charge transformation capabilities of the NPs.

Another type of $\mathrm{pH}$-responsive size and charge transformable NPs was prepared and programmed by overcoming the delivery barrier for improved chemo-ido immunotherapy (Dai et al., 2020). The $\mathrm{pH}$-responsive detachment of DMMA-PEG from a prodrug micelle converts its negative charge of $-5.2 \mathrm{mV}$ to a strongly positive charge of $20 \mathrm{mV}$ and reduces its size from $85 \mathrm{~nm}$ to $42 \mathrm{~nm}$. The transformed micelles, with reduced size and positive surface charge, enhance tumor penetration and improve endocytosis efficiency; they also synergistically inhibit melanoma growth through the combined effects of chemotherapy-induced antitumor immune response and IDO-blockade immunotherapy. The $\mathrm{pH}$-responsive morphological switching of peptide-cyanine nanostructures, self-assembled by peptide melittin (MEL), and a NIR-absorbing photothermal cyanine dye, Cypate, which is further coated with an HA polymer for tumor targeting (termed MEL/Cypate@HA), has recently been reported (Figure 2B) (Jia et al., 2019). In an acidic TME, the MEL/Cypate@HA nanosphere, which transforms into net-like nanofibers, affords an inhibitory 
A
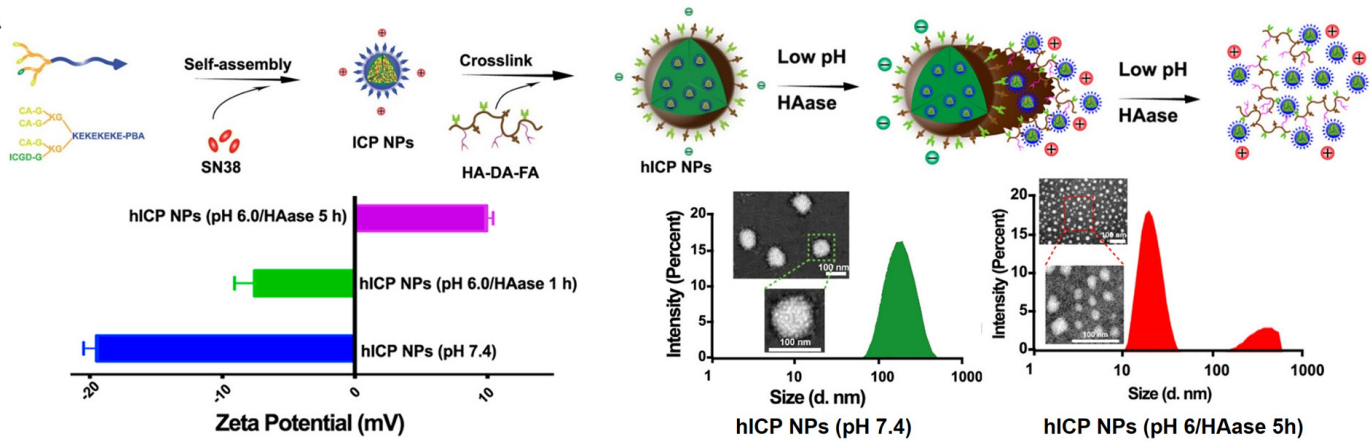

B

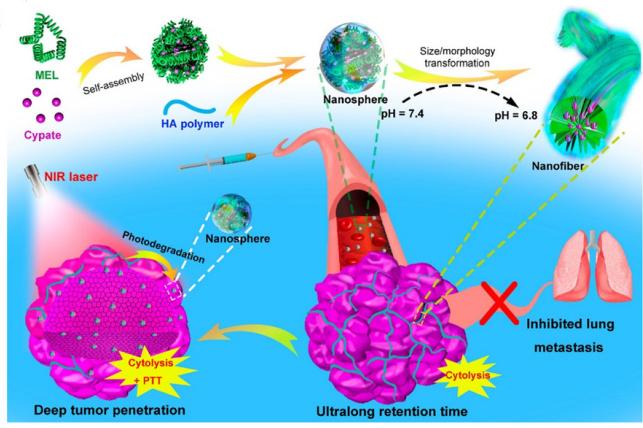

hICP NPs (pH 7.4)

hICP NPs (pH 6/HAase 5h)

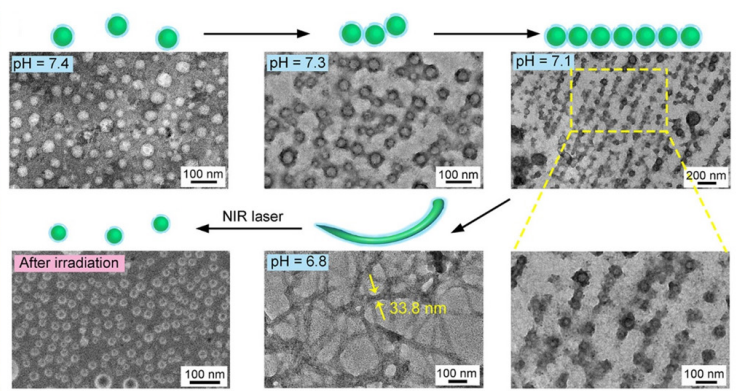

FIGURE 2 | (A) pH-responsive charge and size convertible hICP NPs. Reproduced with permission from reference (Cong et al., 2020). Copyright 2020, American Chemical Society. (B) pH-responsive morphological transformation of MEL/Cypate@HA nanospheres followed by their photodegradation into small nanospheres. Reproduced with permission from reference (Jia et al., 2019). Copyright 2019, American Chemical Society.

effect on tumor cell mobility and also increases its tumor retention time. The $\mathrm{pH}$-responsive peptide sequence in MEL was found to be responsible for the morphological transformation. The nanofibers were photodegraded into small nanospheres (25 nm) via NIR laser irradiation during the cypate-mediated PTT, which further improved deep tumor penetration of the loaded MEL and the MEL-induced cytolysis.

\section{pH-RESPONSIVE PNBS FOR TUMOR IMAGING}

Imaging of heterogeneous tumors is essential for early detection, diagnoses, and precise treatment monitoring. The unique acidic characteristic of tumors enables the design of PNBS with a $\mathrm{pH}$-responsive amplification of the signal-to-noise $(\mathrm{S} / \mathrm{N})$ ratio during tumor-specific imaging (Feng L. et al., 2018; Yang et al., 2020). Thus far, numerous $\mathrm{pH}$-responsive smart PNBS have been reported for in vivo cancer imaging using techniques such as MRI, PAI, FI, and USI (Li Y. et al., 2016; Liu et al., 2017). In this section, we describe recent reports of $\mathrm{pH}$-responsive imaging signal amplification in tumors and ratiometric imaging of $\mathrm{pH}$ in tumors.

\section{pH-Responsive MRI}

Non-invasive imaging techniques, such as MRI, provide a high spatial resolution and detailed three-dimensional anatomical images (Kircher and Willmann, 2012). During MRI, the magnetic field introduced by powerful magnets forces protons in the body to align with the magnetic field. A radiofrequency current is then pulsed through the patient, which stimulates the protons to spin out of equilibrium, causing strain against the pull of the magnetic field. Once the radiofrequency field is switched off, the protons relax back or realign with the magnetic field by releasing energy, which is detected using MRI sensors. The proton relaxation time, as well as the energy released during this process, depends on the chemical nature of the protons and their environments. Longitudinal or spin-lattice relaxation time $\left(T_{1}\right)$ and transversal $\left(T_{2}\right)$ or spin-spin relaxation times of protons are involved in MRI, where $T_{2} \leq T_{1}$. The relaxation times $\left(T_{1}\right.$ or $\left.T_{2}\right)$ of water protons in lesions can be sped up using an MRI contrast agent. The $T_{1}$ and $T_{2}$ contrast agents increase the $\mathrm{S} / \mathrm{N}$ ratios or the contrast of the lesion for more accurate diagnoses of cancer-related pathological diseases. Therefore, the delivery of MR contrast agents to the tumor by using tumor acidity to improve the $\mathrm{S} / \mathrm{N}$ of imaging has received significant research attention.

For example, a $T_{1}$ (longitudinal) $\mathrm{MR}$ contrast agent, releasing PNBS composed of $\mathrm{MnO}_{2}$, has been extensively investigated for its ability to release $\mathrm{Mn}^{2+}$ ions in acidic tumors (Cai et al., 2019). Mi et al. (2016) prepared $\mathrm{Mn}^{2+}$-doped calcium phosphate (CaP) NPs for the $\mathrm{pH}$-responsive MRI contrast on-off system for signal amplification in subcutaneous C26 tumorbearing mice. CaP NPs were prepared via the mineralization of poly(ethylene glycol)- $b$-poly(glutamic acid) [PEG-b-P(Glu)] block copolymers with $\mathrm{Mn}^{2+}, \mathrm{Ca}^{2+}$, and $\mathrm{HPO}_{42}^{-}$in the aqueous phase, followed by hydrothermal treatment to improve the 

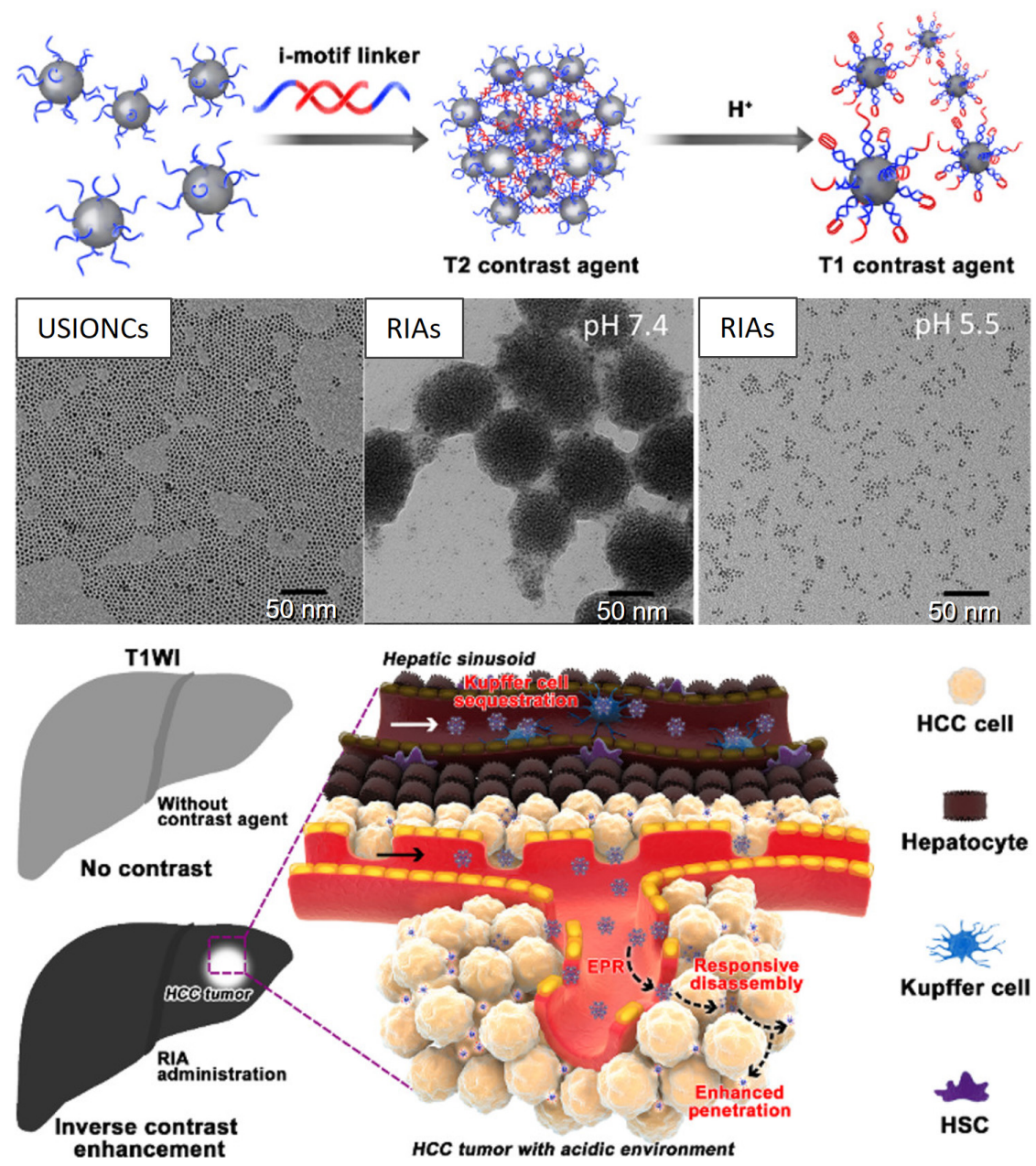

FIGURE 3 | pH-responsive conversion of the MRI contrast agent confirmed via TEM images, and used for inverse contrast enhancement for the detection of small hepatocellular carcinomas (HCCs). Reproduced with permission from reference (Lu et al., 2018). Copyright 2018, American Chemical Society.

mechanical strength of the CaP matrix. The PEG shell in the resulting PEGMnCaP helped maintain the size of the NPs at $\sim 60 \mathrm{~nm}$ by preventing further expansion of the $\mathrm{CaP}$ core. The CaP core of accumulated PEGMnCaP NPs was dissolved in the acidic TME, thereby releasing $\mathrm{Mn}^{2+}$ ions. This $\mathrm{Mn}^{2+}$ readily interacted with surrounding proteins and slowly rotated the $\mathrm{Mn}$ protein system, which enhanced the sharp contrast due to the increased $T_{1}$ relaxivity of $\mathrm{Mn}^{2+}$. Additionally, the PEGMnCaP NPs rapidly and selectively brightened and detected hypoxic regions in solid tumors and detected invisible millimeter-sized metastatic tumors in the liver, owing to their $\mathrm{pH}$-responsive signal amplification capabilities. According to another report, a $T_{2}$ contrast agent, such as $\mathrm{Fe}_{3} \mathrm{O}_{4} \mathrm{NPs}$, was loaded in the hydrophobic core of a $\mathrm{pH}$-sensitive polymer (PEG-poly $(\beta$-amino ester) (PAE) micelle and was released and exposed to water molecules at the acidic tumor. Furthermore, a shortened water proton relaxation time $T_{2}$ responsible for contrast enhancement during MRI was reported (Gao et al., 2010). Recently, a $\mathrm{pH}$-responsive conversion of i-motif DNA-assisted iron oxide nanocluster assemblies (RIAs) from a $T_{2}$ to a $T_{1}$ contrast agent for the detection of small hepatocellular carcinomas (HCCs) has been reported (Figure 3) (Lu et al., 2018). The i-Motif DNAs exhibit $\mathrm{pH}$-responsive structural transformation from single-stranded to intercalated quadruple-helical structures. In the acidic TME, the $T_{2}$ contrast agent RIAs (120 nm), composed of the $T_{1}$ contrast agent ultrasmall iron oxide nanoclusters (USIONCs), is disassembled back to USIONCs. The $\mathrm{pH}$ dependent detachment of the i-Motif cross-linker is responsible for this intelligent contrast agent transformation, resulting in bright HCCs and a healthy dark liver owing to the inverse contrast-enhancing imaging mode transformation.

\section{pH-Responsive USI}

Ultrasound imaging is portable, cost-effective, and enables realtime imaging. Despite these advantages, the low contrast in USI decreases its sensitivity, thereby limiting its diagnostic applications. Thus, contrast-enhanced US (CEUS), which utilizes $1-10-\mu \mathrm{m}$ microbubbles (MBs) as the contrast agent, has been developed (Sun and Emelianov, 2019). However, these MBs cannot accumulate at disease sites via the EPR effect owing to 

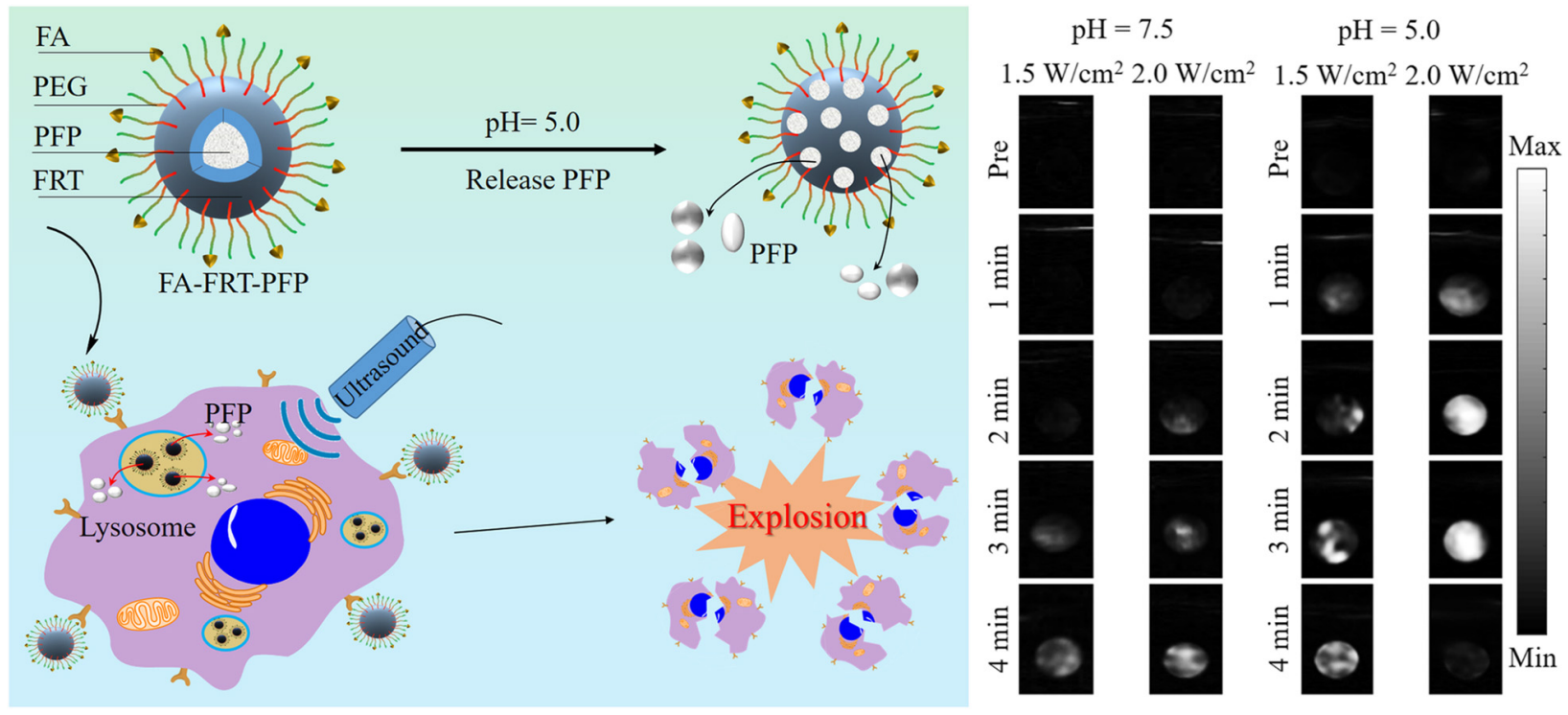

FIGURE 4 | pH-responsive USI using PFP releasing FA-FRT-PFP nanoprobes, which shows enhanced USI contrast at acidic pH. Reproduced with permission from reference (Li et al., 2020). Copyright 2020, Springer.

their large sizes, limiting their application in vivo. Thus, the size of contrast agents should be in the nanometer range in order to accumulate at disease sites. Therefore, reducing the size of MBs and transforming them into nanobubbles (NBs) appears as a straightforward solution. However, NBs have low stability and low echogenicity, and they are excessively small to efficiently scatter ultrasonic waves at the frequencies used in clinical applications. Gas-generating NPs are introduced as a contrast agent for US imaging to overcome these issues and generate sufficient USI contrast while maintaining the nanometer-scale size.

$\mathrm{pH}$-sensitive release of carbon dioxide $\left(\mathrm{CO}_{2}\right)$ gas from mineralized calcium carbonate polymeric NPs (Min et al., 2015; Kim et al., 2018; Chen et al., 2019) or carbonate side chains containing polymer NPs (Kang et al., 2010) has been reported. These NPs exhibited strong echogenic signals with excellent echo persistence in tumoral acidic $\mathrm{pH}$ by producing $\mathrm{CO}_{2}$ bubbles. Recently, Li et al. (2020) prepared a US therapeutic agent that encapsulates low boiling $\left(\mathrm{bp}=29^{\circ} \mathrm{C}\right.$ ) perfluoropentane $(\mathrm{PFP})$ into $\mathrm{pH}$-sensitive cage protein ferritin (FRT) conjugated with the tumor-targeting molecule FA (FA-FRT-PFP) (Li et al., 2020). FRT disassembles at intracellular $\mathrm{pH}$ and releases PFP. A 3min, low-intensity focused ultrasound (LIFU, $2 \mathrm{~W} / \mathrm{cm}^{2}$ ), which significantly enhances the US signal of the FA-FRT-PFP through the acoustic droplet vaporization (ADV) effect, is produced by the PFP (Figure 4). Additionally, the long duration (4 min) of LIFU irradiation at the lysosomal $\mathrm{pH}$ produced a physical shock wave due to the explosive release of PFP from the FA-FRT-PFP; this subsequently results in effective tumor cell destruction via necrosis. Another example of $\mathrm{pH}$-responsive gas $\left(\mathrm{O}_{2}\right)$ generation for USI is the use of manganese dioxide $\left(\mathrm{MnO}_{2}\right)$ NPs that can aptly regulate TME oxygenation owing to their hydrogen peroxidase properties and favorable behavior of breaking-up in mildly acidic and $\mathrm{H}_{2} \mathrm{O}_{2}$-rich TMEs (Gao et al., 2017). The oxygen content in the tumor is elevated to $2.25 \pm 0.07$ times that in the absence of $\mathrm{MnO}_{2}$, which demonstrates its potential for USI.

\section{pH-Responsive PAI}

Photoacoustic imaging is an emerging hybrid modality that combines optical excitation with ultrasonic detection. It allows for deeper tissue penetration and higher spatial resolution compared to traditional optical imaging techniques, owing to the involvement of deep tissue-penetrating ultrasound modalities (Miao and $\mathrm{Pu}, 2018$ ). Endogenous (such as hemoglobin and melanin) and exogenous (such as NIR-emitting dyes, metallic NPs, carbon nanotubes, prophysomes, and 2D graphene analogs) PAI agents have been used for imaging applications. The PA signal depends on the difference in concentration between the healthy and diseased tissues. The poor accumulation and uptake of PAI agents could result in a poor $\mathrm{S} / \mathrm{N}$ ratio in a complex pathological environment. Therefore, activatable PNBS that undergo intrinsic PAI signal amplification only in response to tumor-specific stimuli, including $\mathrm{pH}$, and maintain a dormant state in the absence of $\mathrm{pH}$ can provide a high signal-to-noise ratio at the acidic tumor site. $\mathrm{pH}$-sensitive activation provides a real-time correlation between PNBS states (activated vs. dormant) in pathological processes at the molecular level. This $\mathrm{pH}$-responsive contrast enhancement has garnered significant research attention in recent years.

Miao et al. (2016) developed activated ultrasmall semiconducting oligomer nanoprobes (SONs) with amplified signals for the in vivo PAI of $\mathrm{pH}$. The SONs are prepared via nanoprecipitation of a low-molecular-weight semiconducting oligomer (SO:F-DTS) as the inert PA matrix, doped with a $\mathrm{pH}$ indicator dye, boron-dipyrromethene ( $\mathrm{pH}-\mathrm{BDP})$, and served 
A

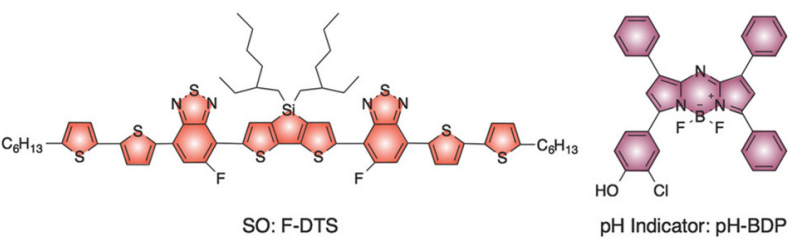

$\mathrm{pH}$ Indicator: $\mathrm{pH}-\mathrm{BDP}$

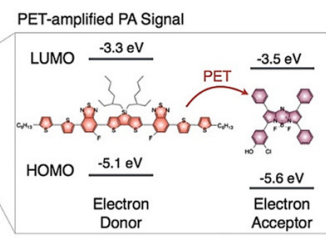

B

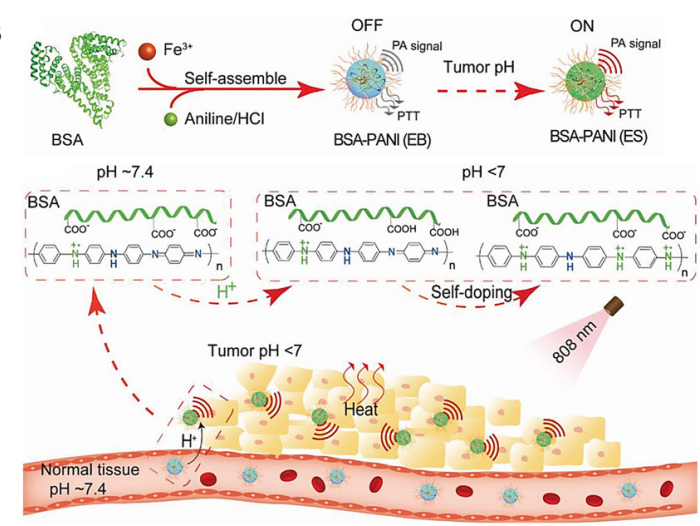

FIGURE 5 | (A) Nanoprobe design of PET-amplified PA imaging of pH. Reproduced with permission from reference (Miao et al., 2016). Copyright 2016, Wiley-VCH GmbH. (B) pH-responsive amplification of PAl using BSA-PANI nanoprobe. Reproduced with permission from reference (Tian et al., 2019). Copyright 2019, Wiley-VCH GmbH.

as a PA enhancer and amphiphilic triblock copolymer (PEG- $b$ PPG- $b$-PEG) as a coprecipitant (Figure 5A). The pH-BDP has a hydroxyl group on its backbone, which undergoes protonation upon acidification and imparts the $\mathrm{pH}$-sensing ability of the SONs. Additionally, the electron affinity and ionization potential of the pH-BDP are lower than those of the SO:F-DTS. Thus, photoinduced electron transfer (PET) is favored between these binary optical partners, which results in the quenched fluorescence of SO:F-DTS; this, in turn, enhances the PA brightness of the SONs. Compared to the $\mathrm{pH}$-inert non-doped SON $\left(\mathrm{SON}_{0}\right)$, the PA brightness of the $50 \mathrm{wt} \% \mathrm{pH}$-BDP-doped nanoprobe $\left(\mathrm{SON}_{50}\right)$ was substantially amplified by a factor of approximately 3.1 at $680 \mathrm{~nm}$. Simultaneously, its ratiometric PA signal $\left(\mathrm{PA}_{680} / \mathrm{PA}_{750}\right)$ increased by approximately 3.1 times on varying the $\mathrm{pH}$ from 7.4 to 5.5 in vitro. Systemic administration of SONs permits non-invasive real-time ratiometric PAI of the $\mathrm{pH}$ with the amplification of brightness in tumors of living mice. Another interesting example of $\mathrm{pH}$-responsive PAI amplification was reported using a polyaniline (PANI)-based theranostic agent composed of bovine serum albumin (BSA) and PANI (Figure 5B) (Tian et al., 2019). The monomer aniline (ANI) polymerizes to PANI in the presence of BSA and $\mathrm{Fe}^{3+}$ ions, and the interaction between hydrophilic BSA and hydrophobic PANI self-assembles to BSA-PANI NPs. The strong BSA-PANI interaction leads to a $\mathrm{pH}$-dependent self-doping effect through intermolecular acid-base reactions between the carboxyl groups of BSA and the imine moieties of the PANI backbone. This increases the degree of protonation of PANI and mediates its transformation from the emeraldine base $(\mathrm{EB})$ to the emeraldine salt (ES) state in an acidic TME $(\mathrm{pH}<7)$. Therefore, in healthy tissues ( $\mathrm{pH} \sim 7.4$ ), the BSA-PANI assemblies exhibit low PAI signals and PTT effects during blood circulation, whereas they exhibited amplified PAI and enhanced PTT in acidic TMEs.

\section{pH-Responsive FI}

Although FI is one of the most widely explored tumor imaging modalities, which also offers the advantages of ease of operation, high sensitivity, and multicolor imaging capabilities, its applications are limited owing to its low depth of light penetration in tissues and poor S/N ratio (Guo et al., 2014; Jin, 2019). The poor S/N in FI is attributed to the non-specific distribution of the imaging probe in tissues and background autofluorescence. $\mathrm{pH}$-responsive fluorescence on-off PNBS are being developed to improve the $\mathrm{S} / \mathrm{N}$ ratio, which can differentiate tumors from their surrounding normal tissue. The FI signal from PNBS is always turned off in the absence of tumorspecific stimuli $(\mathrm{pH})$, providing $\mathrm{pH}$-sensitive augmentation of FI at an acidic TME.

Lower $\mathrm{pH}$ in the TME and overexpressed matrix metalloproteases (MMPs) are two indicators of tumor-associated abnormalities. Quantitative and real-time detection of multiple TME factors through non-invasive multimodality imaging is highly informative. Non-invasive visualizations of these abnormality indicators are an effective approach for studying and confirming abnormal tumor signatures in vivo. A novel target-triggered dual-ratiometric fluorescent nanoprobe for simultaneous mapping of matrix metalloprotease-9 (MMP-9) activity and extracellular in vivo $\mathrm{pH}$ has been reported (Ma et al., 2018). The nanoprobe comprises a ratiometric $\mathrm{pH}$-sensitive dye [( $N$-carboxyhexyl derivative of 3-amino-1,2,4-triazole fused 1,8-naphthalimide), ANNA], its fluorescence is quenched while being attached to the surface of an MRI agent $\left(\mathrm{Fe}_{3} \mathrm{O}_{4} \mathrm{NPs}\right)$ using the peptide substrate of MMP-9, representing the "off" state (Figure 6A). The fluorescence of ANNA was quenched via Förster resonance energy transfer (FRET) between the dye and NPs. However, when the peptide was cleaved by MMP-9, the fluorescence of ANNA was activated ("on" state), allowing for in vivo $\mathrm{pH}$ mapping. This MMP-9 responsive nanoprobe was also co-labeled with a NIR reference dye (Cy5.5), which serves as an always "on" internal reference dye of the resulting secondary ratiometric fluorescent system. The MMP-9 dependent fluorescence emission from ANNA was compared with that of continually emitting Cy5.5 for quantitatively mapping proteases activity across the entire tumor. Extensive imaging studies using these dual-ratiometric probes in a mouse model of human colon cancer revealed 

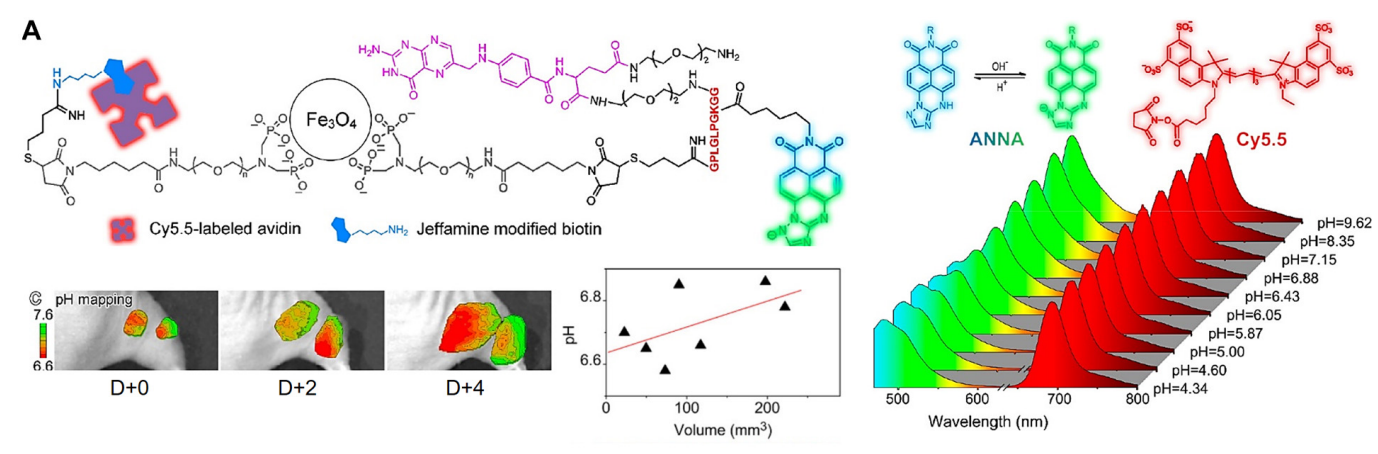

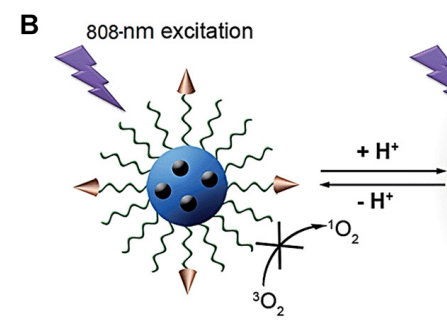

Off state

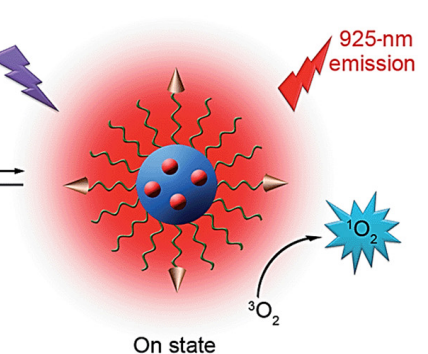

On state

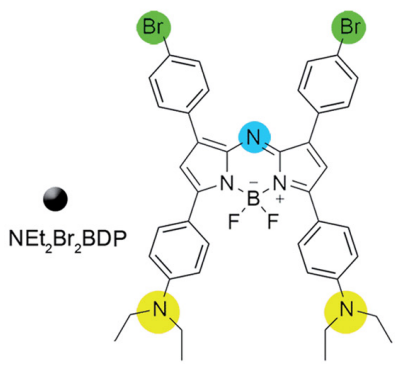

FIGURE 6 | (A) Dual-ratiometric fluorescent nanoprobe for in vivo pH mapping. Protonation-/deprotonation-induced structural transformation of ANNA combined with $\mathrm{pH}$-insensitive $\mathrm{Cy}_{5} .5$ yields two sets of fluorescence spectra recorded at different $\mathrm{pH}$ values excited at 455 and $675 \mathrm{~nm}$, providing ratiometric $\mathrm{pH}$ monitoring capabilities. The intratumorally injected nanoprobes allow for TME pH mapping on different days and TME pH quantification. Reproduced with permission from reference (Ma et al., 2018). Copyright 2018, American Chemical Society. (B) pH-sensitive fluorescence on-off nanoprobe for Fl. Reproduced with permission from reference (Tian et al., 2015). Copyright 2015, Royal Society of Chemistry.

that MMP-9 overexpression and abnormal TME $\mathrm{pH}$ have spatio-temporal correlations. Additionally, the synergistic effect of these two characteristics largely controls the heterogeneous invasion of malignant tumors. Another example is the use of the $\mathrm{pH}$-sensitive fluorescence on-off nanoprobe to achieve highly selective tumor imaging (Tian et al., 2015). A pH-activatable fluorophore with an aza-boron-dipyrromethene structure substituted with diethylaminophenyl and bromophenyl groups $\left(\mathrm{NEt}_{2} \mathrm{Br}_{2} \mathrm{BDP}\right)$ and loaded in a nanomicelle composed of a cyclic RGD peptide-poly(ethylene glycol)-block-poly(lactic acid) (cRGD-PEG-PLA) unimers is used as a FI nanoprobe (Figure 6B). The fluorescence was quenched (off) in the nanomicelle at neutral $\mathrm{pH}(\mathrm{pH}-7.4)$ owing to the photoinduced electron transfer from the diethylaminophenyl moiety to the excited fluorophore. In acidic tumors, the porosity of the nanoprobe increases due to its polymer composition, allowing protons to access the $\mathrm{NEt}_{2}$ groups of the loaded $\mathrm{NEt}_{2} \mathrm{Br}_{2} \mathrm{BDP}$. This protonation increases the fluorescence at $925 \mathrm{~nm}$, resulting in an enhanced, pH-dependent NIR fluorescence (on).

\section{pH-RESPONSIVE TPNBS FOR IMAGE-GUIDED THERAPY}

pH-responsive TPNBS combine the functions of imaging and therapy in a single NP. Their $\mathrm{pH}$-responsiveness selectively and sensitively activates imaging and therapeutic functions in pathological tissues. However, these functions remain inactive in normal tissues. The selective activation of theranostic functions improves the imaging ( $\mathrm{S} / \mathrm{N}$ ratio) and therapeutic efficacy in tumor tissues. Image-guided therapy using TPNBS enables disease detection, monitoring of disease progression, and evaluation of patient response to therapy. ROS-mediated cancer treatment methods, including light-induced PDT (Lucky et al., 2015), US-induced SDT (Qian et al., 2016), and endogenous ROS-induced CDT (Tang et al., 2019), have emerged as potential alternatives to conventional treatment methods such as chemotherapy and surgery, owing to their minimal or non-invasiveness and improved site-specific action. The efficacy of ROS-mediated PDT, SDT, and CDT depends on the ability of the method to produce an elevated level of cytotoxic intracellular ROS. Compared to neutral or less reactive hydrogen peroxide $\left(\mathrm{H}_{2} \mathrm{O}_{2}\right)$ ROS, singlet oxygen $\left({ }^{1} \mathrm{O}_{2}\right)$, peroxy radicals $\left(\bullet \mathrm{O}_{2} \mathrm{H}\right)$, and hydroxyl radicals $(\bullet \mathrm{OH})$ are highly reactive or more cytotoxic to cancer cells. The overproduction of ROS damages lipid bilayers, proteins, and DNA associated with cancer cells and causes cell death (Yao et al., 2005; Trachootham et al., 2009).

\section{pH-Responsive TPNBS in PDT}

In PDT, light excites a photosensitizer from its ground state to a short-lived excited singlet state. The excited photosensitizer either decays back to the ground state by emitting fluorescence or undergoes intersystem crossing to a relatively long-lived triplet excited state with reversed electron spin. In the triplet excited state, the photosensitizer interacts directly with the substrate and cancer cell membrane, leading to proton or electron transfer and the production of radical cations and anions, which further 


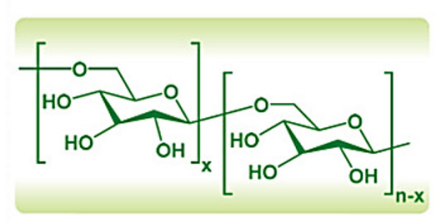

Dextran

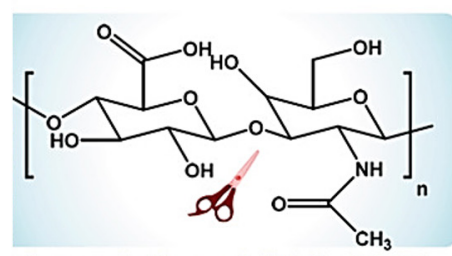

degradation of HA in TME
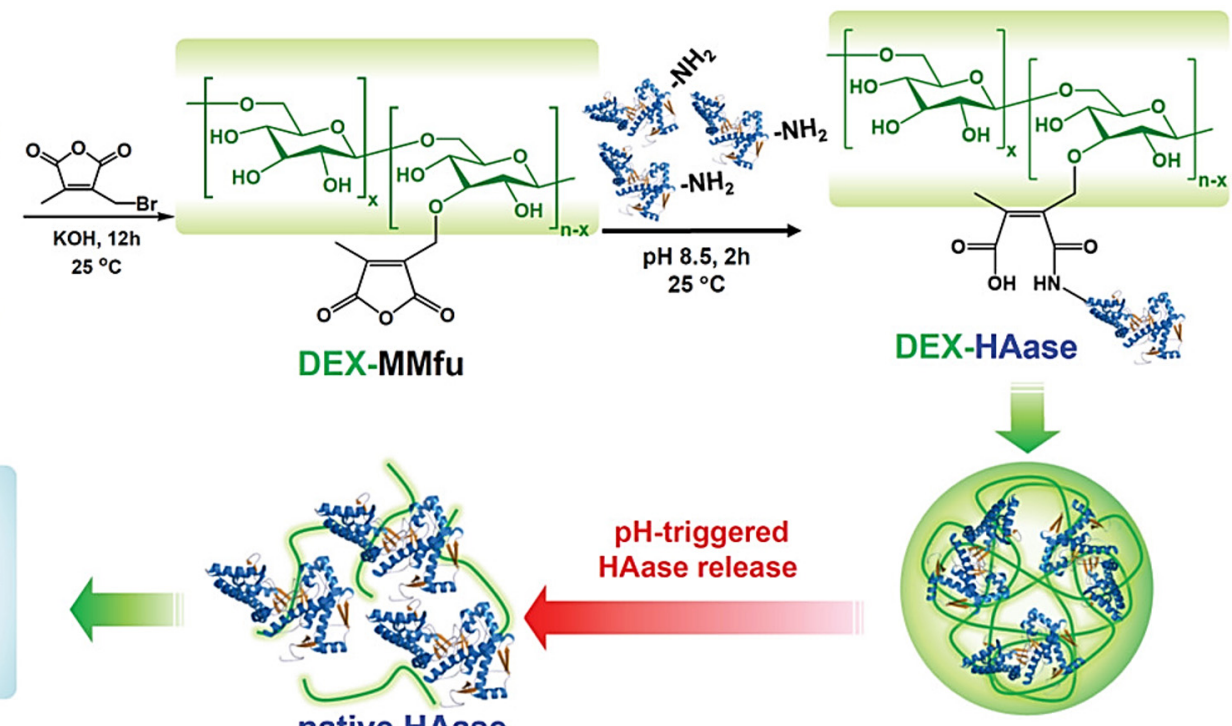

FIGURE 7 | pH-responsive loosening of the condensed extracellular matrix through enzymatic degradation for enhanced tumor permeability of oxygen and nanotheranostics. Reproduced with permission from reference (Wang et al., 2019). Copyright 2019, Wiley-VCH GmbH.

react with oxygen to produce $\mathrm{ROS}$, such as superoxide anion radicals $\left(\mathrm{O}_{2}^{-} \bullet\right), \bullet \mathrm{OH}$, and $\mathrm{H}_{2} \mathrm{O}_{2}$. Alternatively, a triplet excited photosensitizer can directly transfer its energy to molecular oxygen in its ground state $\left({ }^{3} \mathrm{O}_{2}\right)$ and convert it into powerful non-radical ROS, ${ }^{1} \mathrm{O}_{2}$. Therefore, in addition to light and photosensitizers, oxygen is also a key constituent that determines the success of PDT. In poorly oxygenated tumor tissues (tumor hypoxia), the efficacy of PDT is limited (Zhang et al., 2017a). The low depth of penetration of light into the tissue $(<1 \mathrm{~mm})$ is another parameter that limits the application of PDT in deep tumors (Lucky et al., 2015). Tumoral pH-responsive TPNBS were developed to improve the theranostic efficacy of TPNBS for PDT. The $\mathrm{pH}$-responsive TPNBS could augment theranostic efficacy by providing prolonged blood circulation with reduced systemic toxicity, effective tumor accumulation, improved tissue penetration, tumor oxygenation, and tumoral $\mathrm{pH}$-sensitive imaging signal and therapeutic efficacy enhancement.

Qi et al. (2019) reported a pH-responsive simultaneous activation of FI and PDT. Fluorescent dye (Cy7.5), labeled as a $\mathrm{pH}$-responsive copolymer, poly(ethylene glycol)- $b$-poly(2(isopropylamino) ethyl methacrylate) (mPEG- $b$-PDPACy7.5) micelles were encapsulated with a mitochondriatargeted photosensitizer, triphenylphosphonium-conjugated pyropheophorbide-a (TPPa). The resulting NPs were represented as M-TPPa. The fluorescent signal and photoactivity of M-TPPa were completely switched off through the hetero-FRET from TPPa to Cy7.5 molecules. Hetero-FRET facilitated an amplified photodynamic effect in the tumor tissues and suppressed systemic adverse effects to normal tissues. M-TPPa is exposed to the acidic intracellular environment of human HO8910 ovarian cancer cells. The protonation of the tertiary amino groups of its unimers leads to micelle degradation, followed by the efficient early endosomal escape of TPPa owing to its small molecular structure and high permeability across the lipid membrane. Finally, cationic TPPa relocates into the mitochondria to kill the cancer cells through in situ ROS generation upon laser irradiation, providing robust antitumor efficacy with reduced systemic adverse effects. M-TPPa exhibited a 111-fold increase in the fluorescent signal and a 151-fold enhancement in singlet oxygen generation in acidic cancer cells.

In another study, the enzyme HAase was conjugated to a pH-sensitive traceless linker, 3-(bromomethyl)-4-methyl-2,5furandione (MMfu), modified dextran (DEX), DEX-MMfu (Figure 7) (Wang et al., 2019). The obtained DEX-HAase NPs, with diminished immunogenicity compared to free HAase, showed enhanced passive tumor (4T1) accumulation owing to its prolonged blood circulation half-life after intravenous injection. The $\mathrm{pH}$-sensitive release of HAase at the TME loosened the condensed extracellular matrix composed of crosslinked HA due to enzymatic degradation, which enhanced the penetration of oxygen and other therapeutic agents (e.g., liposomes). This improved permeability of the tumor, relieved its hypoxia, and enhanced the therapeutic effect of NP-based PDT, followed by the reversal of the immunosuppressive TME to boost cancer immunotherapy. PAI was used to monitor tumor oxygenation (oxygenated hemoglobin). Liposomes loaded with a photosensitizer, chlorine e6 (Ce6), were used as model nanotherapeutics to demonstrate enhanced PDT and immunotherapy as a result of the $\mathrm{pH}$-sensitive augmentation in tumor permeability.

\section{pH-Responsive TPNBS in SDT}

In SDT, US-induced cavitation causes sonoluminescence or pyrolysis, which contributes to the production of ROS from sonosensitizers once it meets oxygen or water at the tumor tissues (Deepagan et al., 2016; Gong et al., 2019). Depending on the 

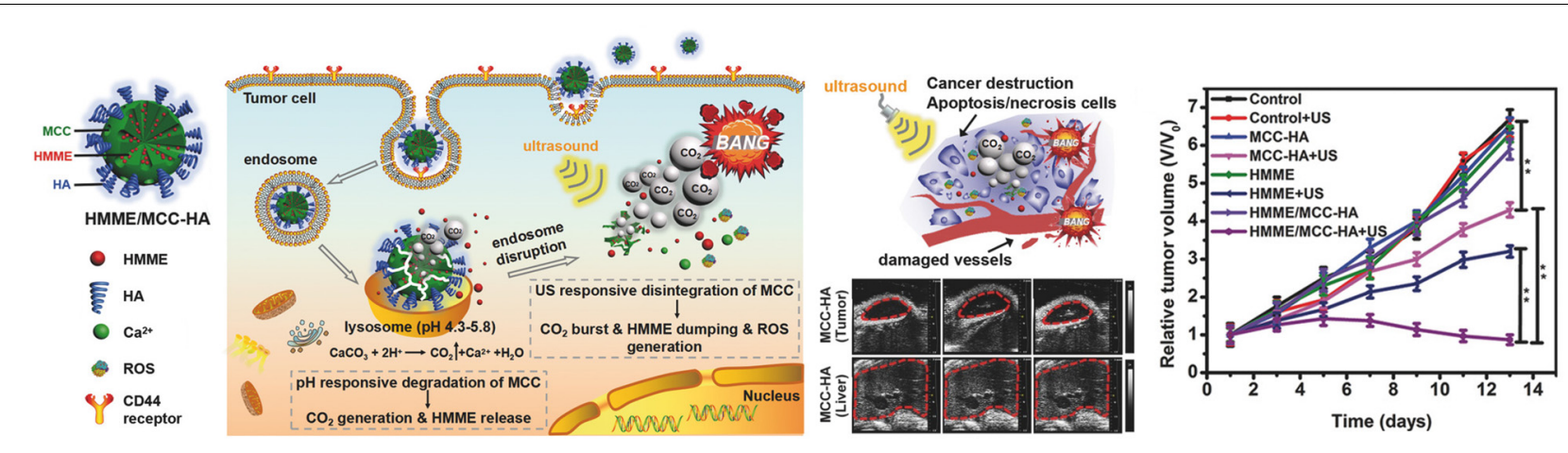

FIGURE 8 | CD44 receptor-mediated endocytosis of HMME/MCC-HA in a cancer cell, followed by the $\mathrm{pH} /$ ultrasound dual-responsive decomposition of $\mathrm{MCC}$, CO 2 bubbling, HMME release, and ROS generation. The $\mathrm{CO}_{2}$ burst release causes cancer destruction via cell apoptosis/necrosis and blood vessel destruction. In vivo therapeutic efficacy of NPs in MCF-7 tumor-bearing mice confirms effective tumor suppression. Compared to liver lesions with neutral pH, acidic tumor tissues exhibit US contrast enhancement, confirming the pH-responsive augmentation of USI. Reproduced with permission from reference (Feng Q. et al., 2018). Copyright 2018, Wiley-VCH GmbH.

frequency, US can be tightly focused, with a penetration of up to several tens of millimeters into soft tissue. Thus, SDT allows access to deeper-seated tumors than PDT (Bailey et al., 2003; Costley et al., 2015). PDT can only be used to treat superficial tumors or endoscopically reachable tumors such as skin cancer, owing to the low penetration depth of light. In PDT treatment, patients must avoid exposure to sunlight after being administered the photosensitizer to avoid light-induced toxicity. Owing to these limitations of PDT, in recent years, research on SDTbased cancer treatment has advanced more rapidly than that on PDT. The lack of an efficient sonosensitizer and poor quantum yield for ROS generation in existing sonosensitizer NPs (Qian et al., 2016) are the primary limitations of SDT. However, these limitations were overcome by developing stimuli-responsive TPNBS (Zhu et al., 2018; Gong et al., 2019). Particularly, a pHresponsive TPNBS for SDT would significantly negate potential side effects in normal tissues and enhance the theranostic efficacy in diseased tissues by providing $\mathrm{pH}$-responsive augmentations in tumor oxygenation, tumor accumulation, tumor penetration, ROS generation, and the $\mathrm{S} / \mathrm{N}$ ratio of imaging signals.

Feng Q. et al. (2018) reported the generation of mesoporous calcium carbonate (MCC) NPs for USI-guided cancer cell necrosis and apoptosis. MCC NPs were loaded with a sonosensitizer [hematoporphyrin monomethyl ether (HMME)], and their surface was decorated with CD44 receptors targeting HA. HA also served as the gatekeeper for the loaded HMME (Figure 8). After receptor-mediated endocytosis, the HMME/MCC-HA decomposed instantaneously via the costimulation of inherent tumoral acidic $\mathrm{pH}$ and US irradiation, followed by simultaneous HMME release and $\mathrm{CO}_{2}$ generation. The degradation associated with the increase in osmotic pressure leads to the redistribution of TPNBS from the endosome to the cytoplasm. In addition to the endosomal escape, the bubbling and bursting of $\mathrm{CO}_{2}$ under the US stimulus results in cavitationmediated irreversible cell necrosis as well as the destruction of blood vessels to further obstruct blood supply, enabling a "bystander effect." The generation of ROS from HMME, triggered by US, causes cell apoptosis via SDT. Therefore, the same $\mathrm{pH}$-responsive TPNBS are used for apoptosis and necrosis and to promote cell injury in a complementary manner. The generated $\mathrm{CO}_{2}$ having the echogenic property, is an effective US contrast agent for the identification of cancerous tissues.

Manganese oxide $\left(\mathrm{MnO}_{\mathrm{x}}\right) \mathrm{NPs}$ can effectively regulate TME oxygenation owing to their hydrogen peroxidase properties and favorable breakup behavior in mildly acidic and $\mathrm{H}_{2} \mathrm{O}_{2}$ rich TMEs (Zhu et al., 2016). Additionally, $\mathrm{MnO}_{\mathrm{x}}$ can breakup via an antioxidant (GSH) depletion reaction in mildly acidic pH (Deng et al., 2011); both of these pathways release $\mathrm{Mn}^{2+}$ ions, which is a T1-MRI agent. The combination of a sonosensitizer and $\mathrm{MnOx}$ can afford MRI-guided augmented SDT due to tumor oxygenation at a mildly acidic TME (Zhu et al., 2018). Similarly, via GSH depletion, sonosensitizer$\mathrm{MnO}_{\mathrm{x}}$ ensures the MRI-guided augmentation of SDT due to the depletion of ROS scavenging GSH in acidic cancer cells, resulting in the enhancement of intracellular oxidative stress (Lin et al., 2020).

\section{pH-Responsive TPNBS in CDT}

Chemodynamic therapy is a unique method for endogenously generating highly cytotoxic ROS $(\bullet \mathrm{OH})$ from TME-rich neutral ROS $\left(\mathrm{H}_{2} \mathrm{O}_{2}\right)$ through a transition metal-ion catalyzed Fentonlike reaction (Chen and Schopfer, 1999; Tang et al., 2019). The released transition metal ions $\left[\mathrm{Fe}^{2+}\right.$ (Li W.-P. et al., 2016), $\mathrm{Mn}^{2+}$ (Lin et al., 2020), $\mathrm{Ti}^{3+}$ (Wang et al., 2020), $\mathrm{Cu}^{+}$(Ma et al., 2019), etc.] from pH-responsive degradable NPs can be used as CDT agents. Most importantly, as CDT involves ROS activation, compared with SDT or PDT, ROS generation via this process is independent of tumor oxygenation. However, novel TPNBS that follow oxygen-dependent CDT via a light-independent PDT mechanism at an acidic tumoral $\mathrm{pH}$ have been recently reported (Lu et al., 2020).

Tumoral pH-responsive theranostic NPs are being developed for imaging guided CDT. For example, a size-controllable, biodegradable, and metastable $\gamma$-phase $\mathrm{MnS}$ nanotheranostic (MnS@BSA) was prepared using BSA as a template for tumor $\mathrm{pH}$-responsive $T_{1}$-weighted MRI-guided integrated CDT and gas 

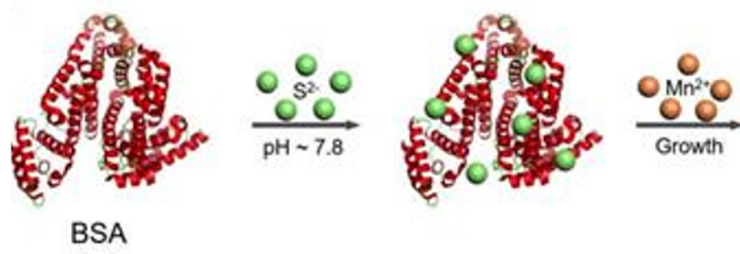

$$
\begin{gathered}
\mathrm{MnS@BSA}+2 \mathrm{H}^{+} \longrightarrow \mathrm{Mn}^{2+}+\mathrm{H}_{2} \mathrm{~S}+\mathrm{BSA} \\
\mathrm{H}_{2} \mathrm{O}_{2} \stackrel{\mathrm{Mn}^{2+} / \mathrm{HCO}_{3}^{-}}{\longrightarrow} \cdot \mathrm{OH}
\end{gathered}
$$
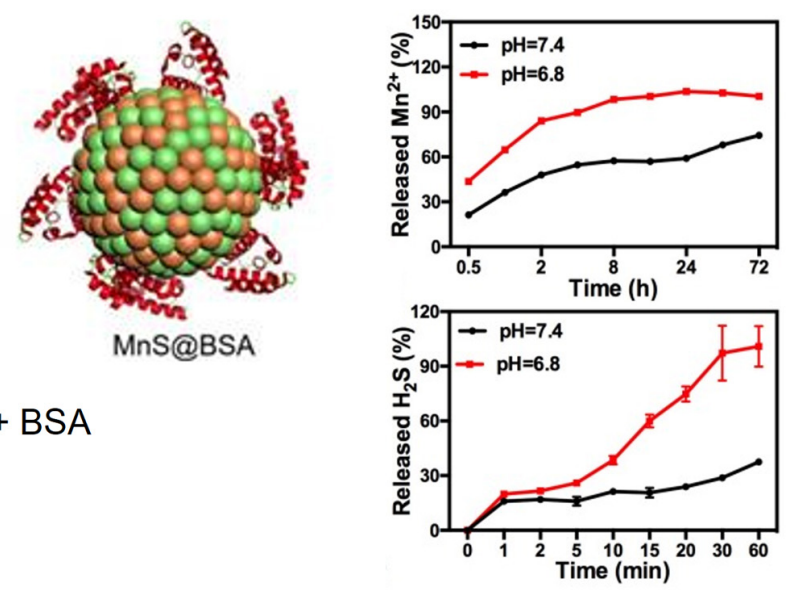

FIGURE 9 | pH-responsive MnS@BSA nanotheranostic for MRI-guided CDT and gas therapy. MnS@BSA enables a pH-responsive release of a CDT agent, Mn²+, and $\mathrm{H}_{2} \mathrm{~S}$ gas. Reproduced with permission from reference (He et al., 2020). Copyright 2020, Wiley-VCH GmbH.

therapy (Figure 9) (He et al., 2020). BSA was used to regulate the size of MnS NPs by tuning the ratio of the proportion of BSA to that of $\mathrm{Mn}^{2+}$. In an acidic TME, the as-prepared MnS@BSA underwent $\mathrm{pH}$-responsive dissociation to simultaneously release the MRI or chemodynamic agent $\left(\mathrm{Mn}^{2+}\right)$ and $\mathrm{H}_{2} \mathrm{~S}$ gas. The anticancer effect of $\mathrm{H}_{2} \mathrm{~S}$ gas, together with the ROS production due to CDT, enables image-guided cancer therapy in 4T1luciferase mammary tumor xenograft bearing $\mathrm{BALB} / \mathrm{c}$ mice with significant suppression of tumor growth. Similarly, a $\mathrm{pH}$ activatable chemodynamic system [ultrathin Mn-oxides $\mathrm{MnO}_{\mathrm{x}}$ nanosheet $\left.\left(\mathrm{Mn}^{\mathrm{II}}\right)_{1}\left(\mathrm{Mn}^{\mathrm{III}}\right)_{3.4}\left(\mathrm{Mn}^{\mathrm{IV}}\right)_{2.8} \mathrm{O}_{11.7}\right]$ that can produce ${ }^{1} \mathrm{O} 2$ under the trigger of acidity through a novel chemodynamic process has recently been developed ( $\mathrm{Lu}$ et al., 2020). The ${ }^{1} \mathrm{O} 2$ produced from $\mathrm{MnO}_{\mathrm{x}}$ using this light-free process chemically reacts with thiophene units of semiconducting polymer (SP) NPs (SPNs); thus, the excited SPNs emit near-infrared (NIR) chemiluminescence through a process called chemical electron exchange luminescence (CIEEL). This process further amplifies the yield of ${ }^{1} \mathrm{O} 2$ generation. In an acidic tumor environment, the MnOx-SPN system achieved satisfactory chemodynamic therapeutic outcomes in vitro and in vivo (4T1 female Balb/c mice). The output of ${ }^{1} \mathrm{O} 2$ generation was calibrated using ratiometric imaging of chemiluminescence/fluorescence, which provides more accurate in situ monitoring of the chemodynamic treatment process.

\section{CONCLUSION AND OUTLOOK}

Owing to their unique physicochemical characteristics, $\mathrm{pH}$ responsive NPs have been extensively investigated for cancer theranostics. The NPs, which exhibit $\mathrm{pH}$-responsive behaviors such as charge conversion, size reduction, and shape changes, would facilitate theranostic efficacy via enhanced tumor accumulation, deep penetration, and/or cellular internalization. In this review, we summarized recent developments that demonstrated how the tumoral $\mathrm{pH}$ could be utilized in the design of intelligent NPs with the potential to enhance noninvasive cancer therapy, disease detection/diagnosis, and their combinations.

By utilizing the unique acidic characteristics of tumors, PNBS with $\mathrm{pH}$-responsive amplification of the $\mathrm{S} / \mathrm{N}$ ratio during tumor-specific imaging has been developed. Thus far, various $\mathrm{pH}$-responsive smart PNBSs have been reported for in vivo cancer imaging using suitable techniques such as MRI, USI, PAI, and FI. MRI is a well-established non-invasive wholebody imaging technique owing to the deep tissue penetration of radiofrequency waves. A pH-responsive PNBS for MRI facilitates a tumoral $\mathrm{pH}$-sensitive release of MRI contrast agents, enabling contrast enhancement of deep-seated tumors during diagnosis and treatment. USI is also available for deep-seated tumor imaging owing to the high tissue penetration depth of US. For instance, pH-responsive gas-generating PNBS with strong echogenic signals and excellent echo persistence has been prepared as the potential USI contrast agent. PAI is an emerging hybrid modality that combines optical excitation with ultrasonic detection. It offers deeper tissue penetration and higher spatial resolution than FI, ascribed to US involvement. PAI using a $\mathrm{pH}$-responsive PNBS would allow for semi-quantitative in vivo $\mathrm{pH}$ sensing and mapping within tumors. Compared to clinically available MRI and USI, the PAI technique would be particularly suitable for in vivo $\mathrm{pH}$ imaging in small animal models to meet basic and preclinical research needs. In vivo applications of FI have limited by the low tissue penetration of light involved, making this approach unsuitable for deep-seated tumor imaging. Similar to PNBS for PAI, fluorescent PNBS are applied to quantitative detection of tumoral $\mathrm{pH}$ and tumor imaging in small animal models.

The TPNBS are intelligent, multifunctional nanosystems that combine the imaging and therapy functions into a single NP. Their $\mathrm{pH}$-responsiveness affords selective activation of theranostic functions in pathological tissues, while being inactive in normal tissues. The selective activation of theranostic functions provides improved imaging ( $\mathrm{S} / \mathrm{N}$ ratio) and therapeutic 
efficacy in acidic tumors with reduced systemic toxicity. Image-guided therapy using TPNBS enables disease detection, monitoring disease progression, and evaluating patients' responses to treatments. Numerous TPNBS with excellent theranostic functions have been reported recently in basic and preclinical research. These designs need to be relatively simple and biocompatible; most of them, however, are excessively complicated for their clinical translation. Until now, the tumor acidity has been mainly used for developing cancer theranostics. It should be emphasized that the tumor acidity plays a crucial role in tumor initiation, progression, and metastasis. Therefore, developing novel nanotheranostics that modulate tumor acidity would be a promising approach to overcome treatment resistance associated with the current TPNBS.

\section{REFERENCES}

Alexis, F., Pridgen, E., Molnar, L. K., and Farokhzad, O. C. (2008). Factors affecting the clearance and biodistribution of polymeric nanoparticles. Mol. Pharm. 5, 505-515. doi: 10.1021/mp800051m

Alsehli, M. (2020). Polymeric nanocarriers as stimuli-responsive systems for targeted tumor (cancer) therapy: recent advances in drug delivery. Saudi Pharm. J. 28, 255-265. doi: 10.1016/j.jsps.2020.01.004

Bailey, M., Khokhlova, V., Sapozhnikov, O., Kargl, S., and Crum, L. (2003). Physical mechanisms of the therapeutic effect of ultrasound (a review). Acoust. Phys. 49, 369-388. doi: 10.1134/1.1591291

Bazban-Shotorbani, S., Hasani-Sadrabadi, M. M., Karkhaneh, A., Serpooshan, V., Jacob, K. I., Moshaverinia, A., et al. (2017). Revisiting structureproperty relationship of $\mathrm{pH}$-responsive polymers for drug delivery applications. J. Control. Release 253, 46-63. doi: 10.1016/j.jconrel.2017.02.021

Blanco, E., Shen, H., and Ferrari, M. (2015). Principles of nanoparticle design for overcoming biological barriers to drug delivery. Nat. Biotechnol. 33:941. doi: $10.1038 /$ nbt.3330

Cabral, H., Matsumoto, Y., Mizuno, K., Chen, Q., Murakami, M., Kimura, M., et al. (2011). Accumulation of sub-100 nm polymeric micelles in poorly permeable tumours depends on size. Nat. Nanotechnol. 6:815. doi: 10.1038/nnano. 2011.166

Cai, X., Zhu, Q., Zeng, Y., Zeng, Q., Chen, X., and Zhan, Y. (2019). Manganese oxide nanoparticles as MRI contrast agents in tumor multimodal imaging and therapy. Int. J. Nanomed. 14, 8321-8344. doi: 10.2147/IJN.S218085

Cao, J., Gao, X., Cheng, M., Niu, X., Li, X., Zhang, Y., et al. (2019). Reversible shielding between dual ligands for enhanced tumor accumulation of $\mathrm{ZnPc}$ loaded micelles. Nano Lett. 19, 1665-1674. doi: 10.1021/acs.nanolett.8b04645

Chaffer, C. L., and Weinberg, R. A. (2011). A perspective on cancer cell metastasis. Science 331, 1559-1564. doi: 10.1126/science.1203543

Chen, S., Xu, X.-L., Zhou, B., Tian, J., Luo, B.-M., and Zhang, L.-M. (2019). Acidic $\mathrm{pH}$-activated gas-generating nanoparticles with pullulan decorating for hepatoma-targeted ultrasound imaging. ACS Appl. Mater. Interfaces 11, 22194-22205. doi: 10.1021/acsami.9b06745

Chen, S. X., and Schopfer, P. (1999). Hydroxyl-radical production in physiological reactions: a novel function of peroxidase. Eur. J. Biochem. 260, 726-735. doi: 10.1046/j.1432-1327.1999.00199.x

Cho, E. C., Xie, J., Wurm, P. A., and Xia, Y. (2009). Understanding the role of surface charges in cellular adsorption versus internalization by selectively removing gold nanoparticles on the cell surface with a I2/KI etchant. Nano Lett. 9, 1080-1084. doi: 10.1021/nl803487r

Cong, Z., Zhang, L., Ma, S.-Q., Lam, K. S., Yang, F.-F., and Liao, Y.H. (2020). Size-transformable hyaluronan stacked self-assembling peptide nanoparticles for improved transcellular tumor penetration and photo-chemo combination therapy. ACS Nano 14, 1958-1970. doi: 10.1021/acsnano.9b0 8434

Costley, D., Mc Ewan, C., Fowley, C., Mchale, A. P., Atchison, J., Nomikou, N., et al. (2015). Treating cancer with sonodynamic therapy: a review. Int. J. Hyperthermia 31, 107-117. doi: 10.3109/02656736.2014.992484

\section{AUTHOR CONTRIBUTIONS}

EP, WU, and JP reviewed and evaluated the literature and wrote the manuscript. All the authors contributed to the article and approved the submitted version.

\section{FUNDING}

This research was funded by the Basic Science Research Program (Grant Numbers 2018R1D1A1B07050867 and 2018R1A2B3006080) and the Korean Research Fellowship (Grant Number 2016H1D3A1909300) of the National Research Foundation (NRF), South Korea.

Couzin, J. (2008). Can fasting blunt chemotherapy's debilitating side effects? Science 321, 1146-1147. doi: 10.1126/science.321.5893.1146a

Da Poian, A., Bacha, T., and Luz, M. (2010). Nutrient utilization in humans: metabolic pathways. Nat. Educ. 3:11.

Dai, L., Li, X., Yao, M., Niu, P., Yuan, X., Li, K., et al. (2020). Programmable prodrug micelle with size-shrinkage and charge-reversal for chemotherapy-improved IDO immunotherapy. Biomaterials 241:119901. doi: 10.1016/j.biomaterials. 2020.119901

Danhier, F., Feron, O., and Préat, V. (2010). To exploit the tumor microenvironment: passive and active tumor targeting of nanocarriers for anti-cancer drug delivery. J. Control. Release 148, 135-146. doi: 10.1016/j.jconrel.2010.08.027

Deepagan, V., Thambi, T., Ko, H., Kang, Y. M., and Park, J. H. (2013). Amphiphilic polysialic acid derivatives: synthesis, characterization, and in-vitro cytotoxicity. J. Nanosci. Nanotechnol. 13, 7312-7318. doi: 10.1166/jnn.2013.8089

Deepagan, V. G., You, D. G., Um, W., Ko, H., Kwon, S., Choi, K. Y., et al. (2016). Long-circulating Au-TiO2 nanocomposite as a sonosensitizer for ROSmediated eradication of cancer. Nano Lett. 16, 6257-6264. doi: 10.1021/acs. nanolett.6b02547

Deirram, N., Zhang, C., Kermaniyan, S. S., Johnston, A. P., and Such, G. K. (2019). pH-responsive polymer nanoparticles for drug delivery. Macromol. Rapid Commun. 40:1800917. doi: 10.1002/marc.201800917

Deng, R., Xie, X., Vendrell, M., Chang, Y.-T., and Liu, X. (2011). Intracellular glutathione detection using $\mathrm{MnO} 2$-nanosheet-modified upconversion nanoparticles. J. Am. Chem. Soc. 133, 20168-20171. doi: 10.1021/ja2100774

Dong, Z., Feng, L., Zhu, W., Sun, X., Gao, M., Zhao, H., et al. (2016). $\mathrm{CaCO} 3$ nanoparticles as an ultra-sensitive tumor-pH-responsive nanoplatform enabling real-time drug release monitoring and cancer combination therapy. Biomaterials 110, 60-70. doi: 10.1016/j.biomaterials.2016.09.025

Fam, S. Y., Chee, C. F., Yong, C. Y., Ho, K. L., Mariatulqabtiah, A. R., and Tan, W. S. (2020). Stealth coating of nanoparticles in drug-delivery systems. Nanomaterials 10:787. doi: 10.3390/nano10040787

Feng, L., Dong, Z., Tao, D., Zhang, Y., and Liu, Z. (2018). The acidic tumor microenvironment: a target for smart cancer nano-theranostics. Natl. Sci. Rev. 5, 269-286. doi: 10.1093/nsr/nwx062

Feng, L., Li, K., Shi, X., Gao, M., Liu, J., and Liu, Z. (2014). Smart pHresponsive nanocarriers based on nano-graphene oxide for combined chemoand photothermal therapy overcoming drug resistance. Adv. Healthc. Mater. 3, 1261-1271. doi: 10.1002/adhm.201300549

Feng, Q., Zhang, W., Yang, X., Li, Y., Hao, Y., Zhang, H., et al. (2018). $\mathrm{pH} /$ ultrasound dual-responsive gas generator for ultrasound imaging-guided therapeutic inertial cavitation and sonodynamic therapy. Adv. Healthc. Mater. 7:1700957.

Feng, Q. H., Zhang, W. X., Yang, X. M., Li, Y. Z., Hao, Y. W., Zhang, H. L., et al. (2018). pH/ultrasound dual-responsive gas generator for ultrasound imagingguided therapeutic inertial cavitation and sonodynamic therapy. Adv. Healthc. Mater. 7:1700957. doi: 10.1002/adhm.201700957

Gao, G. H., Im, G. H., Kim, M. S., Lee, J. W., Yang, J., Jeon, H., et al. (2010). Magnetite-nanoparticle-encapsulated $\mathrm{pH}$-responsive polymeric micelle as an 
MRI probe for detecting acidic pathologic areas. Small 6, 1201-1204. doi: $10.1002 / \mathrm{smll} .200902317$

Gao, S., Wang, G., Qin, Z., Wang, X., Zhao, G., Ma, Q., et al. (2017). Oxygen-generating hybrid nanoparticles to enhance fluorescent/photoacoustic/ultrasound imaging guided tumor photodynamic therapy. Biomaterials 112, 324-335. doi: 10.1016/j.biomaterials.2016.10.030

Gong, F., Cheng, L., Yang, N., Betzer, O., Feng, L., Zhou, Q., et al. (2019). Ultrasmall oxygen-deficient bimetallic oxide MnWOX nanoparticles for depletion of endogenous gsh and enhanced sonodynamic cancer therapy. Adv. Mater. 31:1900730. doi: 10.1002/adma.201900730

Gong, F., Yang, N., Wang, X., Zhao, Q., Chen, Q., Liu, Z., et al. (2020). Tumor microenvironment-responsive intelligent nanoplatforms for cancer theranostics. Nano Today 32:100851. doi: 10.1016/j.nantod.2020.100851

Guo, Z., Park, S., Yoon, J., and Shin, I. (2014). Recent progress in the development of near-infrared fluorescent probes for bioimaging applications. Chem. Soc. Rev. 43, 16-29. doi: 10.1039/C3CS60271K

Han, K., Zhang, W. Y., Zhang, J., Lei, Q., Wang, S. B., Liu, J. W., et al. (2016). Acidity-triggered tumor-targeted chimeric peptide for enhanced intra-nuclear photodynamic therapy. Adv. Funct. Mater. 26, 4351-4361. doi: 10.1002/adfm. 201600170

He, T., Qin, X., Jiang, C., Jiang, D., Lei, S., Lin, J., et al. (2020). Tumor pHresponsive metastable-phase manganese sulfide nanotheranostics for traceable hydrogen sulfide gas therapy primed chemodynamic therapy. Theranostics 10:2453. doi: 10.7150/thno.42981

Hoshyar, N., Gray, S., Han, H., and Bao, G. (2016). The effect of nanoparticle size on in vivo pharmacokinetics and cellular interaction. Nanomedicine 11, 673-692. doi: $10.2217 / \mathrm{nnm} .16 .5$

Jaque, D., Martínez Maestro, L., Del Rosal, B., Haro-Gonzalez, P., Benayas, A., Plaza, J. L., et al. (2014). Nanoparticles for photothermal therapies. Nanoscale 6, 9494-9530. doi: 10.1039/C4NR00708E

Jia, H.-R., Zhu, Y.-X., Liu, X., Pan, G.-Y., Gao, G., Sun, W., et al. (2019). Construction of dually responsive nanotransformers with nanospherenanofiber-nanosphere transition for overcoming the size paradox of anticancer nanodrugs. ACS Nano 13, 11781-11792. doi: 10.1021/acsnano.9b05749

Jin, T. (2019). Critical review-recent progress in NIR fluorophores emitting over $1000 \mathrm{~nm}$ for bioimaging. ECS J. Solid State Sci. Technol. 8:R9. doi: 10.1149/2. 0111901jss

Kaittanis, C., Shaffer, T. M., Ogirala, A., Santra, S., Perez, J. M., Chiosis, G., et al. (2014). Environment-responsive nanophores for therapy and treatment monitoring via molecular MRI quenching. Nat. Commun. 5, 1-11. doi: 10.1038/ ncomms 4384

Kakkar, A., Traverso, G., Farokhzad, O. C., Weissleder, R., and Langer, R. (2017). Evolution of macromolecular complexity in drug delivery systems. Nat. Rev. Chem. 1, 1-17. doi: 10.1038/s41570-017-0063

Kanamala, M., Wilson, W. R., Yang, M., Palmer, B. D., and Wu, Z. (2016). Mechanisms and biomaterials in $\mathrm{pH}$-responsive tumour targeted drug delivery: a review. Biomaterials 85, 152-167. doi: 10.1016/j.biomaterials.2016. 01.061

Kang, E., Min, H. S., Lee, J., Han, M. H., Ahn, H. J., Yoon, I.-C., et al. (2010). Nanobubbles from gas-generating polymeric nanoparticles: ultrasound imaging of living subjects. Angew. Chem. Int. Ed. 49, 524-528. doi: 10.1002/anie. 200903841

Kang, H., Hu, S., Cho, M. H., Hong, S. H., Choi, Y., and Choi, H. S. (2018). Theranostic nanosystems for targeted cancer therapy. Nano Today 23, 59-72. doi: 10.1016/j.nantod.2018.11.001

Kang, S., Kim, Y., Song, Y., Choi, J. U., Park, E., Choi, W., et al. (2014). Comparison of $\mathrm{pH}$-sensitive degradability of maleic acid amide derivatives. Bioorgan. Med. Chem. Lett. 24, 2364-2367. doi: 10.1016/j.bmcl.2014.03.057

Kim, H., Sehgal, D., Kucaba, T. A., Ferguson, D. M., Griffith, T. S., and Panyam, J. (2018). Acidic pH-responsive polymer nanoparticles as a TLR7/8 agonist delivery platform for cancer immunotherapy. Nanoscale 10, 20851-20862. doi: 10.1039/C8NR07201A

Kircher, M. F., and Willmann, J. K. (2012). Molecular body imaging: MR imaging, CT, and US. Part I. principles. Radiology 263, 633-643. doi: 10.1148/radiol. 12102394

Kocak, G., Tuncer, C., and Bütün, V. (2017). pH-Responsive polymers. Polymer Chem. 8, 144-176. doi: 10.1039/C6PY01872F
Kong, L., Campbell, F., and Kros, A. (2019). DePEGylation strategies to increase cancer nanomedicine efficacy. Nanoscale Horizons 4, 378-387. doi: 10.1039/ C8NH00417J

Kumar, E. K. P., Feldborg, L. N., Almdal, K., and Andresen, T. L. (2013). Synthesis and characterization of a micelle-based $\mathrm{pH}$ nanosensor with an unprecedented broad measurement range. Chem. Mater. 25, 1496-1501. doi: $10.1021 / \mathrm{cm} 302922 \mathrm{~d}$

Kumar, E. K. P., Søndergaard, R. V., Windschiegl, B., Almdal, K., and Andresen, T. L. (2014). Cross-linked self-assembled micelle based nanosensor for intracellular pH measurements. J. Mater. Chem. B 2, 6652-6659. doi: 10.1039/ C4TB00446A

Lei, Q., Wang, S.-B., Hu, J.-J., Lin, Y.-X., Zhu, C.-H., Rong, L., et al. (2017). Stimuli-responsive "cluster bomb" for programmed tumor therapy. ACS Nano 11, 7201-7214. doi: 10.1021/acsnano.7b03088

Li, H.-J., Du, J.-Z., Liu, J., Du, X.-J., Shen, S., Zhu, Y.-H., et al. (2016). Smart superstructures with ultrahigh $\mathrm{pH}$-sensitivity for targeting acidic tumor microenvironment: instantaneous size switching and improved tumor penetration. ACS Nano 10, 6753-6761. doi: 10.1021/acsnano.6b02326

Li, J., Ji, H., Jing, Y., and Wang, S. (2020). pH-and acoustic-responsive platforms based on perfluoropentane-loaded protein nanoparticles for ovarian tumortargeted ultrasound imaging and therapy. Nanoscale Res. Lett. 15:31. doi: 10. 1186/s11671-020-3252-z

Li, L., Sun, W., Zhong, J., Yang, Q., Zhu, X., Zhou, Z., et al. (2015). Multistage nanovehicle delivery system based on stepwise size reduction and charge reversal for programmed nuclear targeting of systemically administered anticancer drugs. Adv. Funct. Mater. 25, 4101-4113. doi: 10.1002/adfm. 201501248

Li, W.-P., Su, C.-H., Chang, Y.-C., Lin, Y.-J., and Yeh, C.-S. (2016). Ultrasoundinduced reactive oxygen species mediated therapy and imaging using a fenton reaction activable polymersome. ACS Nano 10, 2017-2027. doi: 10.1021/ acsnano. 5 b06175

Li, X., Zheng, B.-Y., Ke, M.-R., Zhang, Y., Huang, J.-D., and Yoon, J. (2017). A tumor-pH-responsive supramolecular photosensitizer for activatable photodynamic therapy with minimal in vivo skin phototoxicity. Theranostics 7:2746. doi: $10.7150 /$ thno. 18861

Li, Y., Yang, H. Y., and Lee, D. S. (2016). Polymer-based and pH-sensitive nanobiosensors for imaging and therapy of acidic pathological areas. Pharm. Res. 33, 2358-2372. doi: 10.1007/s11095-016-1944-y

Lin, X., Liu, S., Zhang, X., Zhu, R., Chen, S., Chen, X., et al. (2020). An ultrasound activated vesicle of janus $\mathrm{Au}-\mathrm{MnO}$ nanoparticles for promoted tumor penetration and sono-chemodynamic therapy of orthotopic liver cancer. Angew. Chem. Int. Ed. 59, 1682-1688. doi: 10.1002/anie.201912768

Ling, D., Park, W., Park, S.-J., Lu, Y., Kim, K. S., Hackett, M. J., et al. (2014). Multifunctional tumor $\mathrm{pH}$-sensitive self-assembled nanoparticles for bimodal imaging and treatment of resistant heterogeneous tumors. J. Am. Chem. Soc. 136, 5647-5655. doi: $10.1021 / \mathrm{ja} 4108287$

Liu, J.-N., Bu, W., and Shi, J. (2017). Chemical design and synthesis of functionalized probes for imaging and treating tumor hypoxia. Chem. Rev. 117, 6160-6224. doi: 10.1021/acs.chemrev.6b00525

Lu, C., Zhang, C., Wang, P., Zhao, Y., Yang, Y., Wang, Y., et al. (2020). Lightfree generation of singlet oxygen through manganese-thiophene nanosystems for $\mathrm{pH}$-responsive chemiluminescence imaging and tumor therapy. Chem 6, 2314-2334. doi: 10.1016/j.chempr.2020.06.024

Lu, J., Sun, J., Li, F., Wang, J., Liu, J., Kim, D., et al. (2018). Highly sensitive diagnosis of small hepatocellular carcinoma using $\mathrm{pH}$-responsive iron oxide nanocluster assemblies. J. Am. Chem. Soc. 140, 10071-10074. doi: 10.1021/jacs. $8 \mathrm{~b} 04169$

Lucky, S. S., Soo, K. C., and Zhang, Y. (2015). Nanoparticles in photodynamic therapy. Chem. Rev. 115, 1990-2042. doi: 10.1021/cr5004198

Ma, B., Wang, S., Liu, F., Zhang, S., Duan, J., Li, Z., et al. (2019). Selfassembled copper-amino acid nanoparticles for in situ glutathione "AND" $\mathrm{H} 2 \mathrm{O} 2$ sequentially triggered chemodynamic therapy. J. Am. Chem. Soc. 141, 849-857. doi: $10.1021 /$ jacs.8b08714

Ma, T., Hou, Y., Zeng, J., Liu, C., Zhang, P., Jing, L., et al. (2018). Dual-ratiometric target-triggered fluorescent probe for simultaneous quantitative visualization of tumor microenvironment protease activity and $\mathrm{pH}$ in Vivo. J. Am. Chem. Soc. 140, 211-218. doi: 10.1021/jacs.7b08900 
Matsumura, Y., and Maeda, H. (1986). A new concept for macromolecular therapeutics in cancer chemotherapy: mechanism of tumoritropic accumulation of proteins and the antitumor agent smancs. Cancer Res. 46, 6387-6392.

Mi, P. (2020). Stimuli-responsive nanocarriers for drug delivery, tumor imaging, therapy and theranostics. Theranostics 10:4557. doi: 10.7150/thno.38069

Mi, P., Kokuryo, D., Cabral, H., Wu, H., Terada, Y., Saga, T., et al. (2016). A pHactivatable nanoparticle with signal-amplification capabilities for non-invasive imaging of tumour malignancy. Nat. Nanotechnol. 11:724. doi: 10.1038/nnano. 2016.72

Miao, Q., Lyu, Y., Ding, D., and Pu, K. (2016). Semiconducting oligomer nanoparticles as an activatable photoacoustic probe with amplified brightness for in vivo imaging of $\mathrm{pH}$. Adv. Mater. 28, 3662-3668. doi: 10.1002/adma. 201505681

Miao, Q., and Pu, K. (2018). Organic semiconducting agents for deep-tissue molecular imaging: second near-infrared fluorescence, self-luminescence, and photoacoustics. Adv. Mater. 30:1801778. doi: 10.1002/adma.201801778

Min, K. H., Min, H. S., Lee, H. J., Park, D. J., Yhee, J. Y., Kim, K., et al. (2015). $\mathrm{pH}$-controlled gas-generating mineralized nanoparticles: a theranostic agent for ultrasound imaging and therapy of cancers. ACS Nano 9, 134-145. doi: $10.1021 / \mathrm{nn} 506210 \mathrm{a}$

Mishra, S., Webster, P., and Davis, M. E. (2004). PEGylation significantly affects cellular uptake and intracellular trafficking of non-viral gene delivery particles. Eur. J. Cell Biol. 83, 97-112. doi: 10.1078/0171-9335-00363

Mura, S., Nicolas, J., and Couvreur, P. (2013). Stimuli-responsive nanocarriers for drug delivery. Nat. Mater. 12, 991-1003. doi: 10.1038/nmat3776

Muthu, M. S., Leong, D. T., Mei, L., and Feng, S.-S. (2014). Nanotheranostics? application and further development of nanomedicine strategies for advanced theranostics. Theranostics 4:660. doi: 10.7150/thno.8698

Nowak, A. K., Robinson, B. W., and Lake, R. A. (2002). Gemcitabine exerts a selective effect on the humoral immune response: implications for combination chemo-immunotherapy. Cancer Res. 62, 2353-2358.

Parodi, A., Rudzinska, M., Leporatti, S., Anissimov, Y., and Zamyatnin, A. A. (2020). Smart nanotheranostics responsive to pathological stimuli. Front. Bioeng. Biotechnol. 8:503. doi: 10.3389/fbioe.2020.00503

Peng, H., Liu, X., Wang, G., Li, M., Bratlie, K. M., Cochran, E., et al. (2015). Polymeric multifunctional nanomaterials for theranostics. J. Mater. Chem. B 3, 6856-6870. doi: 10.1039/C5TB00617A

Peng, N., Yu, H., Yu, W., Yang, M., Chen, H., Zou, T., et al. (2020). Sequential-targeting nanocarriers with $\mathrm{pH}$-controlled charge reversal for enhanced mitochondria-located photodynamic-immunotherapy of cancer. Acta Biomater. 105, 223-238. doi: 10.1016/j.actbio.2020.01.005

Qi, T., Chen, B., Wang, Z., Du, H., Liu, D., Yin, Q., et al. (2019). A pH-Activatable nanoparticle for dual-stage precisely mitochondria-targeted photodynamic anticancer therapy. Biomaterials 213:119219. doi: 10.1016/j.biomaterials.2019. 05.030

Qian, X., Zheng, Y., and Chen, Y. (2016). Micro/nanoparticle-augmented sonodynamic therapy (SDT): breaking the depth shallow of photoactivation. Adv. Mater. 28, 8097-8129. doi: 10.1002/adma.201602012

Rosenblum, D., Joshi, N., Tao, W., Karp, J. M., and Peer, D. (2018). Progress and challenges towards targeted delivery of cancer therapeutics. Nat. Commun. 9, 1-12. doi: 10.1038/s41467-018-03705-y

Schöttler, S., Becker, G., Winzen, S., Steinbach, T., Mohr, K., Landfester, K., et al. (2016). Protein adsorption is required for stealth effect of poly (ethylene glycol)and poly (phosphoester)-coated nanocarriers. Nat. Nanotechnol. 11:372. doi: 10.1038/nnano.2015.330

Siegel, R. L., Miller, K. D., and Jemal, A. (2020). Cancer statistics, 2020. CA Cancer J. Clin. 70, 7-30. doi: 10.3322/caac. 21590

Søndergaard, R. V., Christensen, N. M., Henriksen, J. R., Kumar, E. P., Almdal, K., and Andresen, T. L. (2015). Facing the design challenges of particlebased nanosensors for metabolite quantification in living cells. Chem. Rev. 115, 8344-8378. doi: $10.1021 /$ cr400636x

Sun, I.-C., and Emelianov, S. (2019). Gas-generating nanoparticles for contrastenhanced ultrasound imaging. Nanoscale 11, 16235-16240. doi: 10.1039/ C9NR04471J

Sun, Q., Sun, X., Ma, X., Zhou, Z., Jin, E., Zhang, B., et al. (2014). Integration of nanoassembly functions for an effective delivery cascade for cancer drugs. $A d v$. Mater. 26, 7615-7621. doi: 10.1002/adma.201401554
Swietach, P., Vaughan-Jones, R. D., and Harris, A. L. (2007). Regulation of tumor $\mathrm{pH}$ and the role of carbonic anhydrase 9. Cancer Metastasis Rev. 26, 299-310. doi: 10.1007/s10555-007-9064-0

Tang, Z., Liu, Y., He, M., and Bu, W. (2019). Chemodynamic therapy: tumour microenvironment-mediated Fenton and Fenton-like reactions. Angew. Chem. Int. Ed. 58, 946-956. doi: 10.1002/anie.201805664

Thews, O., Gassner, B., Kelleher, D. K., Schwerd, G., and Gekle, M. (2006). Impact of extracellular acidity on the activity of P-glycoprotein and the cytotoxicity of chemotherapeutic drugs. Neoplasia 8, 143-152. doi: 10.1593/neo.05697

Tian, J., Zhou, J., Shen, Z., Ding, L., Yu, J.-S., and Ju, H. (2015). A pH-activatable and aniline-substituted photosensitizer for near-infrared cancer theranostics. Chem. Sci. 6, 5969-5977. doi: 10.1039/C5SC01721A

Tian, Q., Li, Y., Jiang, S., An, L., Lin, J., Wu, H., et al. (2019). Tumor pHresponsive albumin/polyaniline assemblies for amplified photoacoustic imaging and augmented photothermal therapy. Small 15:1902926. doi: 10.1002/smll. 201902926

Trachootham, D., Alexandre, J., and Huang, P. (2009). Targeting cancer cells by ROS-mediated mechanisms: a radical therapeutic approach? Nat. Rev. Drug Discov. 8, 579-591. doi: 10.1038/nrd2803

Vander Heiden, M. G., Cantley, L. C., and Thompson, C. B. (2009). Understanding the Warburg effect: the metabolic requirements of cell proliferation. Science 324, 1029-1033. doi: 10.1126/science. 1160809

Vaupel, P., and Harrison, L. (2004). Tumor hypoxia: causative factors, compensatory mechanisms, and cellular response. Oncologist 9, 4-9. doi: 10. 1634/theoncologist.9-90005-4

Vidallon, M. L. P., Douek, A. M., Quek, A., Mcliesh, H., Kaslin, J., Tabor, R. F., et al. (2020). Gas-Generating, pH-responsive calcium carbonate hybrid particles with biomimetic coating for contrast-enhanced ultrasound imaging. Particle Particle Syst. Charact. 37:1900471. doi: 10.1002/ppsc.20190 0471

Wang, C., Cheng, L., Liu, Y., Wang, X., Ma, X., Deng, Z., et al. (2013). Imaging-guided $\mathrm{pH}$-sensitive photodynamic therapy using charge reversible upconversion nanoparticles under near-infrared light. Adv. Funct. Mater. 23, 3077-3086. doi: 10.1002/adfm.201202992

Wang, C., Zhao, T., Li, Y., Huang, G., White, M. A., and Gao, J. (2017). Investigation of endosome and lysosome biology by ultra $\mathrm{pH}$-sensitive nanoprobes. Adv. Drug Deliv. Rev. 113, 87-96. doi: 10.1016/j.addr.2016. 08.014

Wang, H., Han, X., Dong, Z., Xu, J., Wang, J., and Liu, Z. (2019). Hyaluronidase with $\mathrm{pH}$-responsive dextran modification as an adjuvant nanomedicine for enhanced photodynamic-immunotherapy of cancer. Adv. Funct. Mater. 29:1902440. doi: 10.1002/adfm.201902440

Wang, J., Mao, W., Lock, L. L., Tang, J., Sui, M., Sun, W., et al. (2015). The role of micelle size in tumor accumulation, penetration, and treatment. ACS Nano 9, 7195-7206. doi: 10.1021/acsnano.5b02017

Wang, X., Niu, D., Wu, Q., Bao, S., Su, T., Liu, X., et al. (2015). Iron oxide/manganese oxide co-loaded hybrid nanogels as $\mathrm{pH}$-responsive magnetic resonance contrast agents. Biomaterials 53, 349-357. doi: 10.1016/j.biomat erials.2015.02.101

Wang, X., Zhong, X., Bai, L., Xu, J., Gong, F., Dong, Z., et al. (2020). Ultrafine titanium monoxide $(\mathrm{TiO} 1+\mathrm{x})$ nanorods for enhanced sonodynamic therapy. J. Am. Chem. Soc. 142, 6527-6537. doi: 10.1021/jacs.9b10228

Wang, Z., Deng, X., Ding, J., Zhou, W., Zheng, X., and Tang, G. (2018). Mechanisms of drug release in $\mathrm{pH}$-sensitive micelles for tumour targeted drug delivery system: a review. Int. J. Pharm. 535, 253-260. doi: 10.1016/j.ijpharm. 2017.11.003

Warburg, O., Wind, F., and Negelein, E. (1927). The metabolism of tumors in the body. J. Gen. Physiol. 8, 519-530. doi: 10.1085/jgp.8.6.519

Webb, B. A., Chimenti, M., Jacobson, M. P., and Barber, D. L. (2011). Dysregulated pH: a perfect storm for cancer progression. Nat. Rev. Cancer 11, 671-677. doi: $10.1038 / \mathrm{nrc} 3110$

Wilhelm, S., Tavares, A. J., Dai, Q., Ohta, S., Audet, J., Dvorak, H. F., et al. (2016). Analysis of nanoparticle delivery to tumours. Nat. Rev. Mater. 1, 1-12. doi: 10.1038/natrevmats.2016.14

Wu, W., Luo, L., Wang, Y., Wu, Q., Dai, H.-B., Li, J.-S., et al. (2018). Endogenous $\mathrm{pH}$-responsive nanoparticles with programmable size changes for targeted tumor therapy and imaging applications. Theranostics 8:3038. doi: 10.7150/ thno.23459 
Yang, G., Xu, L., Xu, J., Zhang, R., Song, G., Chao, Y., et al. (2018). Smart Nanoreactors for $\mathrm{pH}$-responsive tumor homing, mitochondria-targeting, and enhanced photodynamic-immunotherapy of cancer. Nano Lett. 18, 2475-2484. doi: 10.1021/acs.nanolett.8b00040

Yang, H. Y., Jang, M.-S., Li, Y., Fu, Y., Wu, T. P., Lee, J. H., et al. (2019). Hierarchical tumor acidity-responsive self-assembled magnetic nanotheranostics for bimodal bioimaging and photodynamic therapy. J. Control. Release 301, 157-165. doi: 10.1016/j.jconrel.2019.03.019

Yang, H. Y., Li, Y., and Lee, D. S. (2020). Recent advances of pH-induced charge-convertible polymer-mediated inorganic nanoparticles for biomedical applications. Macromol. Rapid Commun. 2020:2000106. doi: 10.1002/marc. 202000106

Yao, D., Shi, W., Gou, Y., Zhou, X., Aw, T. Y., Zhou, Y., et al. (2005). Fatty acidmediated intracellular iron translocation: a synergistic mechanism of oxidative injury. Free Radic. Biol. Med. 39, 1385-1398. doi: 10.1016/j.freeradbiomed. 2005.07.015

You, D. G., Deepagan, V., Um, W., Jeon, S., Son, S., Chang, H., et al. (2016). ROS-generating TiO 2 nanoparticles for non-invasive sonodynamic therapy of cancer. Sci. Rep. 6:23200. doi: 10.1038/srep23200

Yuan, Y. Y., Mao, C. Q., Du, X. J., Du, J. Z., Wang, F., and Wang, J. (2012). Surface charge switchable nanoparticles based on zwitterionic polymer for enhanced drug delivery to tumor. Adv. Mater. 24, 5476-5480. doi: 10.1002/ adma.201202296

Zhang, C., Chen, W. H., Liu, L. H., Qiu, W. X., Yu, W. Y., and Zhang, X. Z. (2017a). An $\mathrm{O} 2$ self-supplementing and reactive-oxygen-species-circulating amplified nanoplatform via $\mathrm{H} 2 \mathrm{O} / \mathrm{H} 2 \mathrm{O} 2$ splitting for tumor imaging and photodynamic therapy. Adv. Funct. Mater. 27:1700626. doi: 10.1002/adfm.201700626
Zhang, C., Ni, D., Liu, Y., Yao, H., Bu, W., and Shi, J. (2017b). Magnesium silicide nanoparticles as a deoxygenation agent for cancer starvation therapy. Nat. Nanotechnol. 12:378. doi: 10.1038/nnano.2016.280

Zhang, M., Chen, X., Li, C., and Shen, X. (2020). Charge-reversal nanocarriers: an emerging paradigm for smart cancer nanomedicine. J. Control. Release 319, 46-62. doi: 10.1016/j.jconrel.2019.12.024

Zhao, P., Zheng, M., Luo, Z., Fan, X., Sheng, Z., Gong, P., et al. (2016). Oxygen nanocarrier for combined cancer therapy: oxygen-boosted ATP-responsive chemotherapy with amplified ROS lethality. Adv. Healthc. Mater. 5, 2161-2167.

Zhu, P., Chen, Y., and Shi, J. (2018). Nanoenzyme-augmented cancer sonodynamic therapy by catalytic tumor oxygenation. ACS Nano 12, 3780-3795. doi: 10. 1021/acsnano.8b00999

Zhu, W., Dong, Z., Fu, T., Liu, J., Chen, Q., Li, Y., et al. (2016). Modulation of hypoxia in solid tumor microenvironment with $\mathrm{MnO} 2$ nanoparticles to enhance photodynamic therapy. Adv. Funct. Mater. 26, 5490-5498. doi: 10. 1002/adfm.201600676

Conflict of Interest: The authors declare that the research was conducted in the absence of any commercial or financial relationships that could be construed as a potential conflict of interest.

Copyright (C) 2020 Pramod Kumar, Um and Park. This is an open-access article distributed under the terms of the Creative Commons Attribution License (CC BY). The use, distribution or reproduction in other forums is permitted, provided the original author(s) and the copyright owner(s) are credited and that the original publication in this journal is cited, in accordance with accepted academic practice. No use, distribution or reproduction is permitted which does not comply with these terms. 